\title{
El anélido Rotularia spirulaea (Lamarck, 1818) (Polychaeta, Serpulidae) de las margas del Eoceno de la Cuenca de Pamplona (Navarra): microestructura, tafonomía y paleoecología
}

\author{
Javier ELORZA ${ }^{I^{*}} \&$ Humberto ASTIBIA ${ }^{2}$
}

\author{
${ }^{1}$ Departamento de Mineralogía y Petrología, Facultad de Ciencia y Tecnología, Universidad del País Vasco/Euskal Herriko \\ Unibertsitatea (UPV/EHU), Apartado de correos 644, 48080 Bilbao. \\ ${ }^{2}$ Departamento de Estratigrafía y Paleontología, Facultad de Ciencia y Tecnología, Universidad del País Vasco/Euskal Herriko \\ Unibertsitatea (UPV/EHU), Apartado de correos 644, 48080 Bilbao. \\ * Corresponding author
}

Elorza, J. \& Astibia, H. 2017. El anélido Rotularia spirulaea (Lamarck, 1818) (Polychaeta, Serpulidae) de las margas del Eoceno de la Cuenca de Pamplona (Navarra): microestructura, tafonomía y paleoecología. [The annelid Rotularia spirulaea (Lamarck, 1818) (Polychaeta, Serpulidae) from the Eocene marls of the Pamplona Basin (Navarre): microstructure, taphonomy and palaeoecology]. Spanish Journal of Palaeontology, 32 (2), 343-366.

Manuscript received 25 February 2017

Manuscript accepted 22 May 2017

(C) Sociedad Española de Paleontología ISSN 2255-0550

\section{RESUMEN}

En este trabajo se reconoce la presencia de numerosos fósiles del anélido serpúlido Rotularia spirulaea (Lamarck, 1818) en las formaciones Areniscas de Ardanatz y Margas de Ilundain del Eoceno (Bartoniense y (?) Priaboniense) de la Cuenca de Pamplona (Navarra). Se estudian las diferentes capas de la microestructura prismática calcítica de su tubo. El tubo está constituido por una capa externa (1) gruesa, compuesta por tres zonas en continuidad entre ellas y otra capa interna (2), de una extrema delgadez y siempre delimitando el lumen. $R$. spirulaea muestra una notable plasticidad ecofenotípica. La morfología trocoespiral, trocoespiralplaniespiral o planiespiral de los ejemplares podría estar relacionada con las características del sustrato -sin cohesión, medianamente cohesionado o cohesionado, respectivamente-, que condicionarían la capacidad de soportar al organismo durante su ontogenia. La disposición del tubo de $R$. spirulaea durante el estadio trocoespiral sería recostada. Superada esta etapa, podía pasar a una forma planiespiral, con la formación de una carena o quilla estabilizadora hasta adquirir, de forma

\begin{abstract}
This work recognizes the presence of numerous fossils of the annelid serpulid Rotularia spirulaea (Lamarck, 1818) in the Ardanatz Sandstone and Ilundain Marl formations from the Eocene (Bartonian and (?) Priabonian) of the Pamplona Basin (Navarra). The different calcitic layers of the prismatic microstructure of its tube are determined. It is formed by two shell layers: 1) a thick outer layer (1) composed of three zones in continuity among them; and, 2) another extremely thin inner layer (2) that delineates the tube cavity (lumen). $R$. spirulaea shells show a remarkable ecophenotypic plasticity. The trochospiral, trochospiral-planispiral and planispiral coiling of the specimens could be related to the characteristics of the substrate -without cohesion, moderately cohesive or cohesive, respectively-, which would condition the ability to support the organism during its ontogeny. The arrangement of the $R$. spirulaea tube during the trochospiral stage would be a stable horizontal resting position. Once this stage had been overcome, it could pass to a planispiral coiling, with the formation of a stabilizing keel to acquire gradually a vertical
\end{abstract}


gradual, una posición vertical dentro del sustrato fangoso. Es a partir de este estadio, cuando se formaría la parte desenrollada del tubo que, a modo de chimenea, sobresaldría parcialmente del sedimento. La cavidad del tubo (lumen) habitualmente está rellenada por sedimento y/o cementada por cristales de calcita con tres etapas reconocibles y con ejemplos de texturas geopetales, que vendrían a apoyar la idea de una posición de vida vertical en la madurez de los individuos.

Palabras clave: Rotularia spirulaea, morfología, tafonomía, autoecología, Paleógeno. position within the muddy substrate. It is from this stage, when the unrolled part of the tube would form a chimneylike structure, that it would be partially overhang from the sediment. The lumen is usually filled by sediment and/or by three distinct stages of calcite crystal growths, and by geopetal textures, which would support the idea of a vertical life position in the maturity stage of individuals.

Keywords: Rotularia spirulaea, morphology, taphonomy, autoecology, Paleogene.

Rotularia spirulaea (Lamarck, 1818) (Polychaeta, Serpulidae) es una especie muy común en el Eoceno de la Cuenca de Aquitania, en el área norpirenaica (Lamarck, 1818; d'Archiac, 1846, 1850; Rouault, 1850; Bouillé, 1873, 1876). De esa región proviene el material tipo de la especie estudiado por Lamarck, inicialmente denominada Serpula spirulaea, y del que se conserva en el Muséum national d'Histoire naturelle de Paris un sintipo, recogido en los alrededores de la ciudad de Bayona (especímen MNHN.F.R06729, coll. Lamarck 2008-14). $R$. spirulaea es también abundante en muchos enclaves del Eoceno marino del área surpirenaica, desde la región de Vic (Bartoniense-Priaboniense, noreste de la Cuenca del Ebro) (Abad, 2001), siguiendo hacia el este por la Cuenca de Jaca, hasta alcanzar la Cuenca de Pamplona y zonas colindantes (Bartoniense y Priaboniense, suroeste de los Pirineos) (Astibia et al., 2016b), de cuyas extensas formaciones margosas, provienen los materiales descritos en este trabajo. $R$. spirulaea es también reconocida en el dominio del Tetis: Italia (Savazzi, 1995; Vinn, 2008), Croacia (Mikuž, 2008), Hungría (Fozy \& Szente, 2014) y Península de Anatolia (Hoşgör \& Okan, 2006).

Las primeras noticias de esta especie en el área surpirenaica provienen del geólogo francés Carez (1881) quien, precisamente, denominó las margas de Pamplona, junto con las de Vic (Cataluña) y las de Jaca y la Canal de Berdún (Aragón), como "Marnes bleues à Serpula spirulaea". Sin embargo, Carez no dice haber encontrado este poliqueto fósil en los afloramientos navarros. Las primeras citas de $R$. spirulaea en las margas de Pamplona (s.l.) provienen de trabajos posteriores; en concreto los de Ruiz de Gaona (1947), Mendizabal \& Ruiz de Gaona (1949) y Ruiz de Gaona \& Colom (1950), en los que además se mencionan por primera vez la existencia de importantes asociaciones macropaleontológicas en las cercanías de la ciudad de Pamplona. Estos autores aportaron un estudio más exhaustivo sobre su contenido en foraminíferos y concluyeron que las margas pertenecían al Bartoniense. $R$. spirulaea también está presente en los niveles del Bartoniense de la cercana área navarra y América del Sur austral (Olivero, com. pers.). 
de la Sierra de Urbasa, existiendo ejemplares de este fósil no publicados en la colección Ruiz de Gaona. La presencia de $R$. spirulaea en la zona de este estudio fue nuevamente constatada por Astibia et al. (2014, 2016b), siendo localmente abundante en niveles margosos de las formaciones denominadas Areniscas de Ardanatz y Margas de Ilundain, en asociación con otros muchos macrofósiles (corales, briozoos, moluscos, equinodermos y otros grupos) (véase apartado 2).

El objetivo central de este trabajo es contribuir a un mejor conocimiento de la paleobiología de $R$. spirulaea en la zona de estudio mediante: 1) reconocimiento en el campo de su distribución y modo de disposición en los afloramientos margosos (soft bottom); 2) descripción de la morfología externa del tubo carbonatado; 3 ) estudio de la microestructura del tubo, mediante microscopía convencional y microscopía electrónica de barrido (MEB); y, 4) estudio tafonómico, centrado en los procesos fosildiagenéticos, tales como las alteraciones de la composición mineralógica de la microestructura del tubo, rellenos y mineralizaciones del lumen, mediante microscopía convencional, difracción de rayos X (DRX) y catodoluminiscencia (CL). A partir de lo reconocido en el campo y los análisis realizados se pretende elaborar una inferencia paleoecológica, en concreto fundamentar una hipótesis sobre la posición de vida de esta especie en el fondo marino fangoso, aspecto este todavía controvertido.

\section{L OC A L IZACIÓN，MATERIALY MÉTODOS}

La zona de estudio se ubica en la zona media de Navarra, en los Pirineos occidentales (Fig. 1a). Durante el Eoceno el área surpirenaica se estructuró como una cuenca de antepaís orientada al noroeste, adyacente a un orógeno en formación situado al norte. Durante la mayor parte del Eoceno la zona permaneció como una estrecha franja de mar entrante desde el Golfo de Vizcaya a unos $35^{\circ} \mathrm{N}$ de paleolatitud. Con el tiempo, los aportes sedimentarios provenientes de la erosión de los Pirineos emergentes fueron rellenando la cuenca. Así, durante el Eoceno medio y superior (Bartoniense y Priaboniense) grandes sistemas sedimentarios fluvio-deltaicos se extendieron por la cuenca de antepaís (Plaziat, 1981; Pujalte et al., 2002; Barnolas et al., 2004).

Esta evolución está bien registrada en la zona de estudio con una serie alternante de unidades litoestratigráficas margosas y arenosas de edades Bartoniense y (?) Priaboniense (Fig. 1b). La parte inferior de esta sucesión está representada por la Formación Areniscas de Ezkaba, correspondiente a un sistema turbidítico de tipo channellevee, alimentado directamente desde el orógeno que se alzaba hacia el norte (Payros et al., 1997). Las formaciones suprayacentes, Areniscas de Ardanatz y Margas de Ilundain, representan principalmente ambientes de frente deltaico y
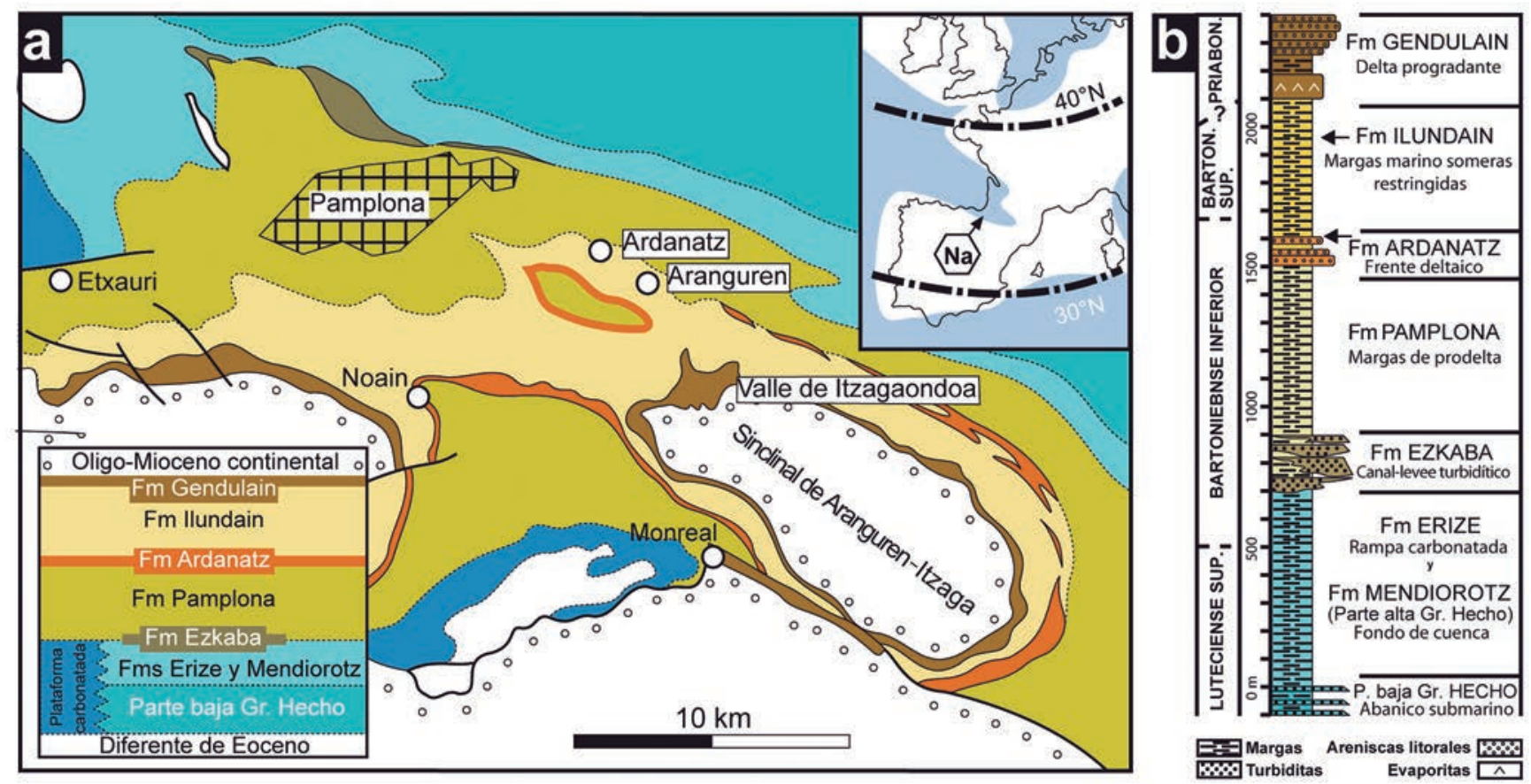

Figura 1. a) Mapa geológico simplificado del área de estudio en la Cuenca de Pamplona y zonas colindantes (Navarra, Pirineos occidentales). Las localidades mencionadas en el trabajo aparecen encuadradas. En la parte superior derecha se muestra un esquema paleogeográfico de Europa occidental durante el Eoceno (Na: Navarra). b) Sección representativa de la sucesión margosa del Eoceno en el área de estudio, con sus unidades litoestratigráficas constituyentes y ambientes deposicionales propuestos en la literatura. Las flechas negras indican la posición estratigráfica aproximada de las zonas de muestreo. (Modificado de Astibia et al., 2016b y Bitner et al., 2016). 
de plataforma restringida (Astibia et al., 2005, 2014). Por último, la formación finieocénica Areniscas de Gendulain se compone de depósitos costeros (Puigdefábregas, 1975). Esta última unidad contiene los depósitos más tardíos de influencia marina de toda la región.

El registro paleoicnológico de estos depósitos es importante y permite caracterizar diferentes icnofacies, indicativas de ambientes que van desde marinos profundos hasta litorales (Payros et al., 2000; Astibia et al., 2016a). El contenido micropaleontológico es abundante, con dominio de los fósiles de foraminíferos y siendo frecuentes también los de ostrácodos. Los macrofósiles corporales son localmente numerosos en las formaciones Areniscas de Ardanatz y Margas de Ilundain. La macrofauna está representada principalmente por fósiles de macroforaminíferos, esponjas hexactinélidas y "litístidas", corales escleractínios, briozoos, braquiópodos, anélidos poliquetos tubícolas, moluscos (gasterópodos, bivalvos, escafópodos y cefalópodos), artrópodos crustáceos, equinodermos (fragmentos de tallo de crinoideos, osículos de asteroideos y espinas y fragmentos de caparazón de equinoideos) y vertebrados (dientes de tiburón, placas de tortuga, y vértebras y costillas de mamíferos sirenios) (Calzada \& Astibia, 1996; Astibia et al., 1999, 2005, 2006, 2014, 2016b; Buffrénil et al., 2008; Bitner et al., 2016).

En las series estudiadas los poliquetos con tubo calcáreo están representados por Rotularia spirulaea y al menos dos especies más de vida libre; una primera de muy pequeña talla, que podría asignarse al género Ditrupa Berkeley, 1835, muy abundante en niveles infrayacentes a los de mayor abundancia de $R$. spirulaea, y una segunda de morfología similar a "Serpula" alata d'Archiac, 1850, especie definida en el Eoceno de Biarritz, en el área norpirenaica. Por otro lado, los tubícolas epibiontes son


Figura 2. a) Laderas acarcavadas (badlands) con formación de un delgado regolito en las margas (tufa, en denominación local) de la transición entre las formaciones Areniscas de Ardanatz y Margas de Ilundain del Bartoniense de Navarra, donde han sido recogidos numerosos ejemplares de Rotularia spirulaea (Lamarck, 1818). b) Aspecto de tres ejemplares sobre estas margas. El de mayor tamaño está bioerosionado por briozoos (? Pinaceocladichnus isp.). c) Ejemplar fragmentado dispuesto vertical en la marga sin alterar descubierta a favor de un reguero. Se considera que este ejemplar adulto está en su posición de vida. d) Detalle de un afloramiento con ejemplares de $R$. spirulaea en la parte alta de la Formación Margas de Ilundain (Bartoniense superior, (?) Priaboniense, Navarra). e) Señales de depredación por gasterópodos (Oichnus isp.) en dos ejemplares (IV.60, arriba, IV.61, abajo) del mismo afloramiento. 
muy abundantes, especialmente sobre conchas de moluscos bivalvos.

La mayoría de los ejemplares de $R$. spirulaea visibles se disponen sueltos sobre el regolito generado a partir de las margas (Figs. 2a-b). Por debajo del regolito se encuentran los lechos de marga buzando ligeramente al sur, dado que pertenecen al flanco norte del Sinclinal de ArangurenItzaga. En algún caso se observa en estos lechos cómo los fósiles de $R$. spirulaea se disponen verticalmente en relación al plano de estratificación de las margas (Fig. 2c) (véase apartado 4).

Para este trabajo han sido recolectados y estudiados un total de 523 fósiles del anélido Rotularia spirulaea (Lamarck, 1818), provenientes principalmente de los niveles margosos AD1.3 (180 ejemplares) y AG1.4 (46 ejemplares), de las secciones AD1 de Ardanatz-Eguesibar y AG1 de Aranguren, en la transición entre las formaciones Areniscas de Ardanatz y Margas de Ilundain (Bartoniense, Navarra, Fig. 1; Astibia et al., 2014, figs. 1 y 2), y de tres localidades del valle de Itzagaondoa (238 ejemplares), en la parte alta de la Formación Margas de Ilundain (Bartoniense superior, (?) Priaboniense) (Fig. 1). El resto de los fósiles (59 ejemplares) provienen de otras secciones de la zona de estudio. La necesidad de protección de estos enclaves, de acuerdo con la legislación en materia de Patrimonio natural y cultural, aconseja no indicar la localización precisa de los mismos. Aquellas personas que deseen más información al respecto pueden ponerse en contacto con los autores de este trabajo.

Para la descripción de los ejemplares se han seguido principalmente los trabajos de Ball (1960), Savazzi (1995), Macellari (1984) y Seilacher et al. (2008). Para el estudio microestructural se ha utilizado la terminología de Ball (1960). Se ha preparado una selección de 27 láminas delgadas de ejemplares de Rotularia en secciones ecuatoriales y transversales, para examinarlas bajo microscopía de luz transmitida mediante una lupa binocular Leica MZ16 equipada con una cámara digital DFC 320, y con un microscopio petrográfico Nikon Labophot T2-Pol acoplado a una cámara Coolpix 4500. Algunas láminas han sido teñidas con alizarina roja S (siguiendo el método de Dickson, 1965). Para un examen por catodoluminiscencia (CL) se ha utilizado un Technosyn Cold Cathode Luminescence system, modelo $8200 \mathrm{Mk}$ II, montado sobre un microscopio Olympus triocular (IGME, Madrid). Dos muestras fueron analizadas por difracción de rayos X (DRX), usando un difractómetro Philips PW-1710 (UPV/EHU). Otras secciones ecuatoriales y transversales han sido empleadas para el examen con el microscopio electrónico de barrido (MEB), utilizando un Jeol JSM-T6400 (UPV/ EHU). Todos los ejemplares estudiados se encuentran depositados provisionalmente en el Departamento de Estratigrafía y Paleontología de la Facultad de Ciencia y Tecnología de la UPV/EHU.

\section{PALEONTOLOGÍA SISTEMÁTICA}

\author{
Clase POLYCHAETA Grube, 1850 \\ Clado CANALIPALPATA Rouse \& Fauchald, 1997 \\ Clado (Orden) SABELLIDA Fauchald, 1977
}

Familia Serpulidae Rafinesque, 1815

Subfamilia Spirorbinae Chamberlin, 1919

Género Rotularia Defrance, 1827

Rotularia spirulaea (Lamarck, 1818)

(Figs. 3-9)

3.1. Sinonimias. Ver Mikuž (2008, p. 162).

\subsection{Descripción}

Morfología externa. $R$. spirulaea genera un tubo biomineralizado de naturaleza carbonatada, cuya forma enrollada en espiral presenta una cierta variabilidad. El inicio o vértice (apex, según Savazzi, 1995) del tubo siempre falta y puede corresponder a una fase inicial de fijación del organismo sobre un sustrato, quizás orgánico y perecedero. En la mayoría de los ejemplares le sigue una porción interna de forma troncocónica, correspondiente a un crecimiento helicoespiral, que pasa a planiespiral involuto, de manera más o menos abrupta, tras las primeras 2-4 vueltas (Figs. 3a-h). La superficie de la espira correspondiente al lado de fijación inicial del vértice del tubo es más bien plana, en tanto que la opuesta tiende a ser ligeramente cóncava. El tubo desarrolla una única carena o quilla periférica, especialmente neta en su parte planiespiral, que presenta secciones sub-triangulares. La quilla no ocupa una posición central sino que está ligeramente desplazada hacia el lado de fijación inicial del vértice del tubo. A veces, la forma troncocónica persiste aparentemente hasta un estado avanzado de crecimiento (conical specimens, según Savazzi, 1995) y la quilla suele formar un resalte basal afilado (Figs. 3j-k, 3r-t). Por el contrario, en otras ocasiones el crecimiento del tubo puede considerarse planiespiral desde el inicio (Fig. 3i). La porción final del tubo en los ejemplares más grandes, pero también a veces en pequeños, suele estar desenrollada, generándose una chimenea (chimney, según Seilacher el al., 2008), formando un ángulo $\left(<45^{\circ}\right)$ con respecto a la porción planiespiral. En la chimenea la quilla primero se reduce y luego desaparece en las cercanías de la abertura (Figs. 3a-b, 3r-t, 3u-ab).

La superficie externa del tubo es bastante lisa, aunque en detalle ofrece finas irregularidades en forma de arrugas y surcos radiales y espirales. En casi todos los ejemplares 



$\mathbf{u}$

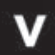

w


Figura 3. Rotularia spirulaea (Lamarck, 1818) del Eoceno medio y (?) superior (Bartoniense y (?) Priaboniense) de la Cuenca de Pamplona (Navarra). Transición entre las formaciones Areniscas de Ardanatz y Margas de Ilundain, secciones de Ardanatz-Eguesibar (AD) y Aranguren (AG) (Astibia et al., 2014). a-b) AD.54, vistas apical-dorsal y umbilical. c-e) AD1.3.15, ejemplar dextrógiro, vistas apical-dorsal, apertural y umbilical. f-h) AD2.2.5, vistas apical-dorsal, apertural y umbilical. i) AD1.3.16, vista apical-dorsal. j-k) AD1.3.13, ejemplar de forma cónica (trocoespiral), vistas apical-dorsal y apertural. l-m) AG1.4.6, vistas apical-dorsal y umbilical. Parte alta de la Formación Margas de Ilundain, sección del valle de Itzagaondoa. n-p) IV.53, vistas apical-dorsal, apertural y umbilical. q) IV.54, vista apical-dorsal. r-t) IV.55, ejemplar dextrógiro de forma cónica, vistas apical-dorsal, apertural y umbilical. u-w) IV.56, vistas apical-dorsal, apertural y umbilical. x) IV.57, vista apical-corsal. y) IV.58, fragmento de la porción desenrollada final del tubo. z-ab) IV.59, vistas apical-dorsal y umbilical. Escala $10 \mathrm{~mm}$. 
estudiados quedan más o menos patentes los surcos o líneas radiales de crecimiento, correspondientes a las sucesivas fases constructivas o incrementos calcáreos (depósito de lamelas), más bien rectas o ligeramente onduladas, dispuestas con cierta regularidad a lo largo de la porción planiespiral del tubo. Suele destacar, así mismo, un neto surco o acanaladura espiral dorsal en ambos lados del tubo, que marca la base de la quilla. En muchos ejemplares se observa también una fina estriación espiral paralela al surco dorsal. Las secciones internas del tubo (lumen) son de forma almendrada (ovaladas afiladas) en la parte cónica del tubo, para hacerse subcirculares afiladas en la porción planiespiral y dar, finalmente, aperturas circulares. La tasa de expansión de la luz interna del tubo (lumen) es pequeña.

De manera análoga a como convencionalmente procede Savazzi (1995), orientados los ejemplares con el vértice hacia arriba, el sentido de enrollamiento de $R$. spirulaea es mayoritariamente levógiro. De un total de 294 ejemplares bien conservados analizados, sólo 12 de ellos (4\%), presentan un sentido de enrollamiento dextrógiro. El diámetro mayor del tubo puede superar los $18 \mathrm{~mm}$ (Tabla 1).

Tabla 1. Dimensiones en milímetros de 456 ejemplares correspondientes a tres poblaciones tafónicas de Rotularia spirulaea (Lamarck, 1818) del Eoceno marino de Navarra; en concreto, dos conjuntos de ejemplares de los niveles AD1.3 y AG1.4, de las secciones AD1 de Ardanatz-Eguesibar y AG1 de Aranguren, respectivamente (Astibia et al. 2014) (transición entre las formaciones Areniscas de Ardanatz y Margas de Ilundain) y un tercer conjunto de ejemplares, de la sección IV del valle de Itzagaondoa (parte alta de la Formación Margas de Ilundain). mín.: valor mínimo; $\bar{x}$ : valor medio; máx. valor máximo; n: número de ejemplares medidos.

\begin{tabular}{|c|c|c|c|c|c|c|c|c|}
\hline \multirow[b]{2}{*}{ Localidad } & \multicolumn{4}{|c|}{ Diámetro } & \multicolumn{4}{|c|}{ Espesor } \\
\hline & mín. & $\bar{x}$ & máx. & $\mathrm{n}$ & mín. & $\bar{x}$ & máx. & $\mathrm{n}$ \\
\hline $\begin{array}{l}\text { AD1.3 (Ardanatz- } \\
\text { Eguesibar) }\end{array}$ & 4.82 & 9.06 & 18.10 & 180 & 1.80 & 2.90 & 4.82 & 180 \\
\hline $\begin{array}{l}\text { AG1.4 } \\
\text { (Aranguren) }\end{array}$ & 4.73 & 9.39 & 15.66 & 46 & 2.15 & 2.88 & 4.81 & 46 \\
\hline IV (Itzagaondoa) & 5.55 & 10.32 & 16.56 & 230 & 2.52 & 3.41 & 5.75 & 230 \\
\hline
\end{tabular}

A los cambios ontogenéticos descritos (crecimiento trocoespiral que pasa a planiespiral y, finalmente, desenrollado) y sus variaciones, cabe añadir que el tubo presenta un crecimiento anisométrico. Los ejemplares de mayor talla tienden a ser más planos, con una mayor relación diámetro/espesor que los de menor tamaño (Fig. 4).

Microestructura. El examen por microscopía convencional de las secciones ecuatoriales y transversales de algunos ejemplares de $R$. spirulaea, recogidos en las formaciones Areniscas de Ardanatz y Margas de Ilundain (Cuenca de Pamplona), permite la diferenciación en el tubo carbonatado de las capas interna y externa que establece Ball (1960) para las especies Rotularia callosa (Stoliczka, 1868) y Rotularia shackletoni (Wilckens, 1910), del Cretácico Superior de la isla de James Ross (Antártida). Así, en los ejemplares objeto de este estudio pueden reconocerse una capa externa gruesa (outer lamellar layer, según Ball, 1960), que a su vez se subdivide en una zona externa (outer zone, e), una zona de transición ( $\mathrm{t}$ ) flexionada y otra zona interna (inner zone, $i$ ) y otra capa interna extremadamente delgada, sin estructura visible (inner structureless layer), que delimita la forma y tamaño del lumen.

Capa externa (1). Como se acaba de indicar, la capa externa (1), bien desarrollada, está constituida por tres zonas reconocibles: una zona interna (i) menos gruesa, en continuidad con una delgada zona de inflexión o transición (t) muy oscura que, a su vez está comunicada con la zona externa (e) de mayor grosor, lo que conforma la superficie exterior del tubo espiral.

Zona interna (i) de la capa externa (1). Destaca por su delgadez en las primeras vueltas de la espira $(<100 \mu \mathrm{m})$, para crecer gradualmente a medida que avanza y llegar a su máximo grosor $(300-500 \mu \mathrm{m})$ en la parte final, donde se produce la porción no enrollada del tubo (Fig. 5a). En esta zona más gruesa se aprecia a ambos lados del lumen una microestructura aparentemente lamelar, con láminas diferenciadas (claras y oscuras), subparalelas entre sí, que presentan un comportamiento asintótico con la capa interna (2) más antigua. También se constata un ahorro de material, al cubrir el espacio separado por un sistema de cámaras vacías mediante el trazado de pilares (p), desde la creciente parte final no enrollada hasta contactar con la zona externa (e) de la vuelta anterior (Fig. 5a). Esta estrategia de formación de pilares (p) también se reproduce en otras partes de la espiral, cuando la zona a rellenar, por la curvatura generada, resulta demasiado grande (Figs. 5b-c).

El supuesto fragmento orgánico, al que se adhiere como anclaje (a) durante su estadio larvario, queda recubierto con una banda micrométrica teñida en tonos rojizos donde no se aprecia su microestructura, aunque bien pudiera corresponder a la capa interna (2) (Figs. 5c-d). Resulta sintomático que el tamaño y forma de la huella que queda del supuesto fragmento orgánico, nunca encontrado, es muy semejante en todos los ejemplares examinados.

En las secciones ecuatoriales cortadas con una cierta oblicuidad puede observarse la zona de transición (t) flexionada más desarrollada entre la zona interna y la externa de la capa externa (1). Ello permite reconocer que existe continuidad entre ambas zonas (i y e) a través de una alternancia de láminas claras y oscuras, dibujando formas sigmoideas con una mayor concentración de 



Figura 4. Diagrama diámetro vs. espesor de la concha en milímetros correspondiente a tres poblaciones tafónicas de Rotularia spirulaea (Lamarck, 1818) del Eoceno marino de Navarra. a) Transición entre las formaciones Areniscas de Ardanatz y Margas de Ilundain, secciones AD1 de Ardanatz-Eguesibar y AG1 de Aranguren (Astibia et al., 2014). ( ): Ejemplares del nivel AD1.3; (घ): ejemplares del nivel AG1.4. b) Parte alta de la Formación Margas de Ilundain. (•): Ejemplares de la sección IV del valle de Itzagaondoa. Nótese el crecimiento anisométrico, señalado por las curvas de regresión.

materia originariamente orgánica (parte oscura) en la zona de transición (t) (Fig. 5d). Ya en secciones próximas al plano ecuatorial, se confirma la nítida continuidad en el crecimiento entre la zona interna y externa mediante la zona de transición, sin que exista ruptura entre ellas (Figs. $5 \mathrm{e}-\mathrm{f})$. Si la concha ha sufrido una deformación temprana, la ligera fracturación que se constata permite reconocer mejor la delgada capa interna (2) que se separa del resto y queda soportada por la masiva cementación en sus dos primeras etapas de $\mathrm{c} 1$ y c2 reconocidas (Fig. $5 \mathrm{~g}$ ). En la zona de separación del tubo enrollado en planiespiral (p.e) para dar paso al crecimiento final no enrollado (n.e) se producen uniones de una cierta complejidad, con contactos directos entre la zona externa más antigua (e) con la zona 
interna más tardía (i*) y la zona externa más antigua (e) con la zona externa más tardía ( $\mathrm{e}^{*}$ ); estos dos últimos complementados por celdas muy pequeñas, ahora ocupadas por calcita (Fig. 5h).

Es reconocible cómo a lo largo del desarrollo de la espira se aprovecha lo ya construido, de tal forma que la progresiva generación de la capa interna (2), es apenas perceptible en la pared interna de la concha (inner wall, según Ball, 1960; fig. 4), y se apoya/construye directamente sobre la zona externa (e) de la capa externa (1) perteneciente a la vuelta anterior. De esta manera, el organismo siempre está en contacto con la delgada capa interna (2), que a su vez se dispone tanto sobre la zona externa existente como en la zona interna en construcción (outer wall e inner wall) (Figs. 6a-c, 7a).

Zona externa (e) de la Capa externa (1). En sección ecuatorial, la zona externa (e) presenta un grosor creciente a lo largo de la espiral, mucho mayor que la zona interna (i), siendo máximo en la parte terminal del tubo enrollado $(\approx 1000 \mu \mathrm{m})$ (Fig. 6). Aparentemente, se mantiene como una masa compacta sin laminación, pero según sea su posición respecto a la espiral, se aprecian las láminas y un contacto de transición ( $\mathrm{t}$ ) extremadamente oscuro que da paso a la zona interna (i), formada por láminas alternantes oscuras y claras ya comentadas anteriormente (Figs. 5d-f). La dirección de la laminación reconocida en la zona externa siempre es contraria a dirección de las láminas de la interna. También se aprecian unas pequeñas formas oscuras (tipo pilares), perpendiculares a la superficie de contacto con la zona interna (Figs. 6ad) que, al ser observados por el MEB, responden a una estructura prismática con tendencia a formar esferulitos (spherulitic prismatic, SphP, según Carter et al., 1990) (Figs. 7a-b). Ya en la parte final, cuando el tubo inicia el paso de la forma espiral a la desenrollada (chimenea), la zona externa resulta ser más gruesa y las laminaciones alternantes (claras y oscuras) son evidentes junto con líneas oscuras perpendiculares a las anteriores, debidas a la microestructura prismática identificada que llegan a la coalescencia (Figs. 8a-b).

En sección transversal, en el espécimen escogido (sección de Ardanatz AD1) se observa cómo el tubo es trocoespiral en las tres primeras vueltas conservadas, para pasar a planiespiral en las dos siguientes (Fig. 8c). Aparentemente, la quilla periférica (carena) se desarrolla a partir de la $4^{\text {a }}$ vuelta de espira, estando formada por la capa externa del tubo (Figs. 8c-h). En la zona de carena, la microestructura de la zona externa (e) de la capa externa (1) dibuja laminaciones claras y oscuras concéntricas entre sí, alcanzando la totalidad de la misma y siempre en contacto con la zona de transición ( $\mathrm{t}$ ) más oscura que da paso a la zona interna (i) (Figs. 8d-g). Debido al mayor desarrollo de la capa externa (1), en estas secciones transversales las zonas interna (i), de transición (t) y externa (e) quedan claramente diferenciadas. Cuando la espira se apoya sobre una superficie carenada anterior (zona externa, e) es visible la formación exclusiva de la imperceptible capa interna (2) para diferenciar el lumen y cómo se extiende lateralmente la capa externa con sus correspondientes zonas i-t-e, cubriendo parcialmente la carena anterior (Figs. 8d-h).

La visión por CL permite observar cambios en la luminiscencia de la zona externa (e) frente a la interna (i) de la llamada capa externa (1). Así, la zona externa es menos luminiscente, aportando tonos rojizos, mientras que la zona interna se une a la zona de transición $(i+t)$, ofreciendo unos colores amarillentos más intensos que resaltan en relación a la falta de luminiscencia del cemento carbonatado c1 y c2, salvo el más tardío c3, que ocupan el lumen (Figs. 9a-d). Resulta de interés apreciar cómo cuando se produce el paso del tubo de enrollado a desenrollado, la nueva zona externa $\left(\mathrm{e}^{*}\right)$ contacta inicialmente con la zona externa (e) de la vuelta anterior mediante pequeños pilares más luminiscentes ( $\mathrm{p}$ ), para seguir avanzando sin que apenas se aprecie el contacto de la nueva zona $\left(\mathrm{e}^{*}\right)$ con la anterior (e) (Figs. 9e-f).

Capa interna (2). La capa interna (2) es considerada como un revestimiento muy delgado, apenas perceptible, en contacto con el lumen, que no presenta estructura visible mediante la microscopía convencional y no siempre queda preservada, posiblemente debido a su inicial composición nacarada aragonítica. Para Savazzi (1995) la capa interna (inner layer) está compuesta de láminas subparalelas a la superficie interna de la concha y es posiblemente de composición aragonítica y/o rica en materia orgánica, estando parcialmente o totalmente neomorfizada a calcita por la alteración diagenética. En nuestro caso, se aprecia esta delgada capa interna como calcítica (2) en los ejemplares examinados mediante el microscopio petrográfico. No alcanza las 20-50 micras de grosor y no es posible determinar su estructura por este medio (Figs. 5a, 5f-g). Si fuera de composición nacarada aragonítica, muy posiblemente correspondería a una "sheet nacreous structure" (Carter et al., 1990). Por otra parte, en el examen mediante CL se observa que esta capa está remarcada con una intensa luminiscencia brillante de tonos amarillos frente a la luminiscencia más apagada de las diferentes zonas de la capa externa (1) (Figs. 9e-h). Las muestras analizadas (dos ejemplares de Ardanatz e Itzagaondoa, respectivamente) mediante difracción de rayos $\mathrm{X}$, no detectan la presencia de aragonito, siendo dominante la calcita con ligeros indicios de cuarzo procedentes del relleno del lumen.

\subsection{Comparaciones}

Morfología externa. La forma del tubo de los ejemplares estudiados se ajusta a la diagnosis original de Lamarck (1818) para "Serpula spirulaea". Los fósiles de 


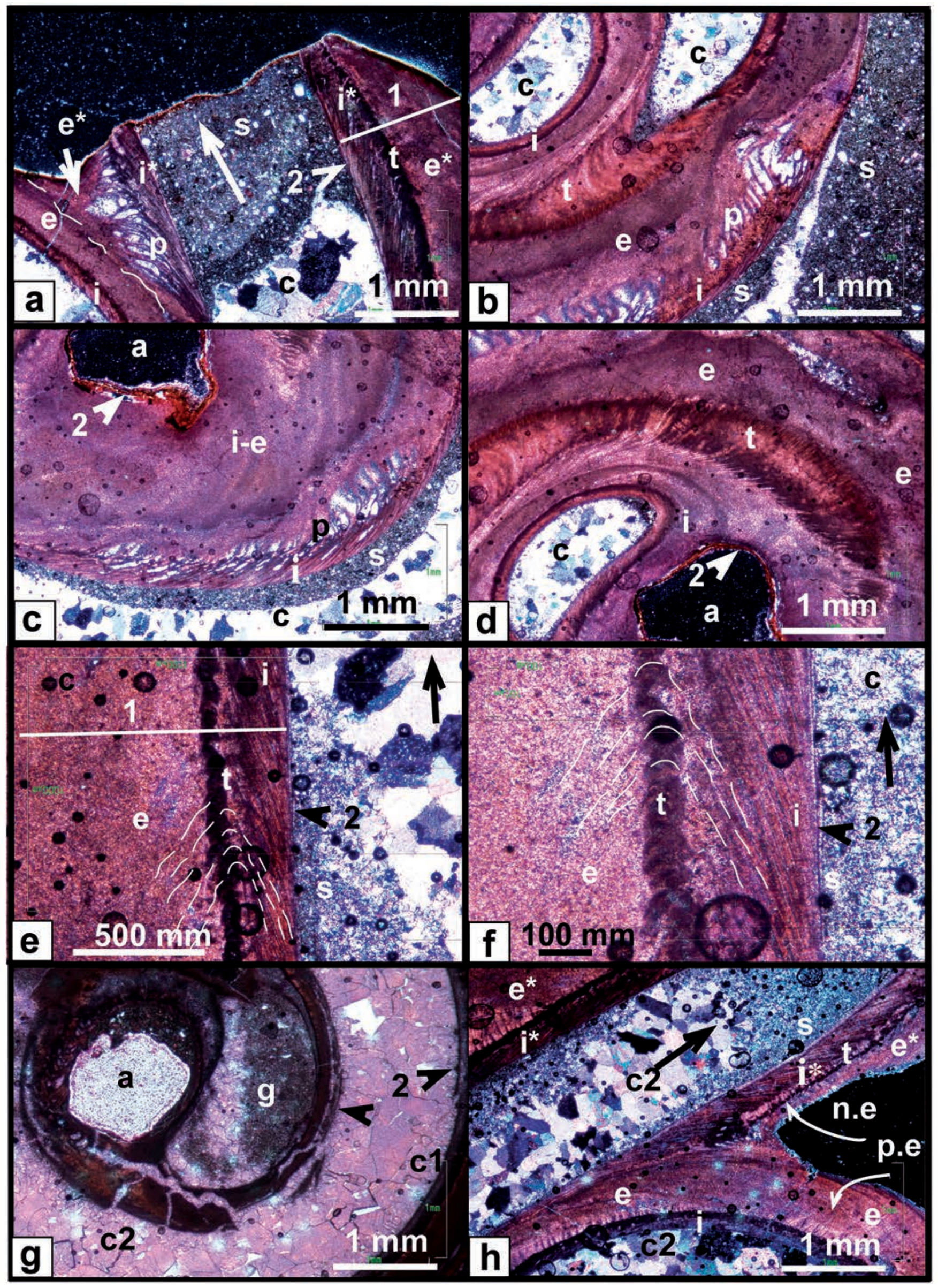


Navarra también encajan tanto morfológicamente como biométricamente con lo indicado por Savazzi (1995) para fósiles de Rotularia spirulaea del Eoceno superior de varias localidades del NE de Italia y también por Hosgör \& Okan (2006), Mikuž (2008) y otros autores, para ejemplares de la misma especie en Turquía y Croacia. Siguiendo a Savazzi (1995), en Rotularia fallax (Wilckens, 1910) (R. (Austrorotularia) fallax en Macellari, 1984) del Maastrichtiense de las islas de Snow Hill y Seymour (Antártida) la superficie del tubo es muy rugosa y la cara sutural está fuertemente excavada, incluso en la parte desenrollada. Además $R$. fallax presenta tres quillas longitudinales bien marcadas, mientras que en $R$. spirulaea hay una sola carena (Seilacher et al., 2008; fig. 15). En Rotularia polygonalis (J. de C. Sowerby, 1829) son dos las crestas periféricas en la parte enrollada del tubo. En Rotularia rugosa (Whitfield, 1890) del Cretácico Superior de Beirut (Líbano), la quilla no es mediana sino cercana al margen del lado libre. Rotularia pseudospirulaea (Oppenheim, 1901) del Eoceno de Austria mantiene una forma ligeramente cónica durante toda la ontogenia, no tiene quilla y presenta dos cordones espirales en cada lado del tubo longitudinal. Esta última característica también está presente en Rotularia leptostoma (Gardner, 1939) del Eoceno de Texas. En otras especies, como Rotularia hisingeri (Lundgren, 1891) y Rotularia saxonica (Müller, 1966) los tubos son bastante lisos y de sección circular, diferentes, por tanto, de los tubos algo rugosos y de secciones subtriangulares carenadas de $R$. spirulaea.
Microestructura. Las descripciones sobre las diferentes microestructuras encontradas en la literatura no son completamente coincidentes entre sí, ni tampoco con lo observado en este trabajo, dado que la interpretación y la terminología cambian de una publicación a otra. Se pueden diferenciar dos etapas cronológicas en la investigación, con textos caracterizados por la utilización de técnicas de estudio diferentes: una primera, donde los trabajos se centran fundamentalmente en la morfología y, ocasionalmente, en el análisis microestructural por medio de la microscopía convencional (Schmidt, 1955; Ball, 1960; Macellari, 1984; Jäger, 1993; Savazzi, 1995; entre otros) y otra etapa más reciente, donde se potencia el estudio del análisis ultraestructural del tubo en diferentes géneros y especies de anélidos mediante MEB, prescindiendo de la microscopía habitual (Vinn, 2005, 2007, 2008; Vinn et al., 2008; Vinn \& Kupriyanova, 2011; Ippolitov et al., 2014; entre otros).

Ball (1960) es quizás el autor que primeramente estableció y remarcó con rotundidad la presencia de las dos diferentes capas formadoras de la microestructura de Rotularia, tal como hemos comentado y seguido en nuestra investigación. Cabe indicar un pequeño error en su apreciación de las dos diferentes capas establecidas (Ball, 1960, figs. 4A-B, p. 22), puesto que su considerada inner zone (b) de la outer lamellar layer (1) perteneciente a la parte de la pared interna (inner wall), debe corresponder a la previa outer zone (a), dado que forma parte de la vuelta anterior como así se confirma con la inclinación de las lamelas dibujadas.

Figura 5. Microestructura del tubo de un ejemplar (IV.48) de Rotularia spirulaea (Lamarck, 1818) en sección ecuatorial, de la sección de Itzagaondoa (Formación Margas de Ilundain, Bartoniense-(?) Priaboniense de Navarra). a) Parte terminal del tubo en espiral e inicio del tubo recto donde se aprecian una zona interna desarrollada (i*), una fina y oscura zona de transición ( $\mathrm{t}$ ) y una zona externa más desarrollada $\left(\mathrm{e}^{*}\right)$, todas ellas pertenecientes a la capa externa (1). La capa interna (2) es apenas perceptible. El lumen queda ocupado parcialmente por sedimento (s), además de cemento de calcita equant (c). Nótese cómo la solución morfo-funcional utilizada para pasar del tubo en espiral a la forma recta es generar a partir de la zona interna (i*) unos pilares $(p)$, ya pertenecientes a la zona externa $\left(\mathrm{e}^{*}\right)$, para enlazar con la zona externa espiralada (e) anterior. Nícoles cruzados. b) Otro ejemplo de la misma sección ecuatorial con la zona interna (i), zona de transición (t) flexionada, zona externa (e) y la cavidad de la última vuelta rellena de sedimento (s), mientras que las cavidades anteriores quedan cementadas por calcita equant (c). En la última vuelta se generan pilares (p) para ocupar el mayor espacio posible. Nícoles cruzados. c) Contacto inicial del organismo con algún tipo partícula orgánica, ahora perdida, utilizada como anclaje (a), con la posible capa interna (2) teñida en rojo. La sección no permite separar con nitidez las zonas interna-externa (ie) entre sí. Son visibles los pilares (p) con las celdas vacías en contacto con la zona interior (i), además del relleno de la cavidad por sedimento (s) y cemento de calcita (c). Nícoles cruzados. d) Zona de transición (t) entre la zona interna (i) y la externa (e), remarcada por un mayor contenido de materia orgánica y con lamelas curvadas en continuidad entre ambas capas. En esta imagen también están presentes la cementación por calcita (c) del lumen y la zona de anclaje inicial (a), remarcado por la capa interna (2). Nícoles cruzados. e-f) Detalles de la capa externa (1) formada por la zona interna (i), zona de transición (t) y la zona externa (e). Las lamelas de las capas dibujan sentidos opuestos, remarcados por las líneas blancas discontinuas. Es visible la delgada capa interna (2). Nícoles cruzados. g) Detalle del anclaje (a) y las primeras vueltas afectadas por una deformación que fractura y separa tempranamente la capa interior (2). El lumen del tubo está ocupado por dos fases de cemento visibles c1 y c2, así como un relleno con microesparita en estructura geopetal (g). h) Zona de "despegue" entre el tubo planiespiral (p.e) y la zona no enrollada (n.e). Aquí se produce el contacto de la zona exterior más antigua (e) con la zona interior más tardía (i*) y se inicia la zona de transición (t), remarcada por la banda oscura entre la zona ( $\left.i^{*}\right)$ y la exterior más tardía $\left(\mathrm{e}^{*}\right)$. Nícoles cruzados. La capa interna (2), apenas perceptible, queda señalada por (2) y las puntas de flechas en las Figuras (5a, 5c-g). Las flechas situadas en el lumen indican la dirección de enrollamiento en las Figuras (5a, 5e-f, 5h). 
Macellari (1984) describe un nuevo subgénero (Austrorotularia) y dos nuevas especies, Rotularia (Austrorotularia) tenuilaevis y Rotularia (Austrorotularia) zinsmeisteri en el Cretácico de las islas Seymour y Snow Hill (Antártida). Además, diferencia y correlaciona mediante Rotularia (Austrorotularia) cuatro zonas diferentes en la potente secuencia estratigráfica estudiada. Observa cómo Rotularia es más abundante en sedimentos de grano fino con un alto porcentaje de fango lo cual es interpretado como una respuesta paleoecológica oportunística. En episodios de rápido incremento poblacional (blooming) reconoce un aumento de tamaño y un decrecimiento en el número de formas trocoespirales. Macellari (1984) considera además que la posición de vida de Rotularia es enterrada verticalmente por debajo de la interfase aguasedimento (ver apartado 5). Savazzi (1995), en su trabajo sobre varios géneros de Rotularia procedentes de diferentes zonas y edades de Europa, Norteamérica y Antártida, sigue principalmente las ideas de Ball (1960) sobre la microestructura y mantiene que el tubo carbonatado del género Rotularia está habitualmente compuesto de una delgada capa o pared interior y otra capa exterior más gruesa.

Vinn (2008) se centra en el estudio de la ultraestructura de Rotularia spirulea (Lamarck, 1818) y $R$. nummularia (Schlotheim, 1820), con detalladas observaciones mediante el MEB, pero sin presentar la visión de un campo más amplio y complementario que ofrece la microscopía convencional de luz transmitida. Así, considera cómo el tubo carbonatado de $R$. spirulaea está compuesto por dos capas distintas pero que en ocasiones son transicionales. La capa exterior (outer layer), de grosores variables $(1,6 \mathrm{~mm}$ dorsalmente y $0,67 \mathrm{~mm}$ lateralmente, medido en la apertura del tubo), tiene una estructura homogénea (según Carter et al., 1990) y está compuesta de clusters orientados de forma variable de cristales angulares de 7-30 $\mu \mathrm{m}$. La capa interna (thin inner tube layer, 0,18 $\mathrm{mm}$ de grosor), con una estructura lamelar-fibrilar (según Carter et al., 1990) está compuesta de prismas alargados (5-8 $\mu \mathrm{m}$ de longitud $\mathrm{y}$ de $1 \mu \mathrm{m}$ de grosor). También reconoce la inclusión de esferulitos prismáticos en la frontera entre la capa exterior y la interior, incrementando la zonación perpendicular a su eje longitudinal. Según Vinn \& Kupriyanova (2011), los tubos de otras especies pueden ofrecer en su microestructura hasta 4 tipos de capas. Algunas de estas capas están constituidas por grandes cristales densamente empaquetados y otras están compuestas de cristales finos escasamente empaquetados. Los tubos con una capa densa exterior (dense outer layer, DOL) producen un suave brillo característico en la superficie del tubo, presumiblemente evolucionado como una adaptación contra la depredación por perforación de gasterópodos y la descomposición de la concha por disolución en aguas profundas insaturadas de carbonato cálcico.

\section{TAFONOMÍA}

\subsection{Alteración tafonómica. Observaciones de campo}

Durante el proceso de sedimentación, que conformó los niveles muestreados en las formaciones Areniscas de Ardanatz y Margas de Ilundain, el sedimento en el fondo marino respondía como un barro blando. La sedimentación pudo haber sufrido interrupciones, llegando a generar pequeñas superficies de condensación, apenas perceptibles, propiciando a su vez una cementación temprana.

Las margas aflorantes conforman abruptas laderas acarcavadas (bandlands), características en las zonas no cubiertas por la vegetación (Figs. 2a-b). La práctica totalidad de los ejemplares de Rotularia spirulaea observados en el campo están desplazados por la erosión y, como ya se ha comentado anteriormente (ver apartado 2), se acumulan sueltos sobre un regolito de escasa potencia $(5-10 \mathrm{~cm})$, con agrietamiento poligonal y estructuras popcorn, generado a partir de las margas. Los fósiles no muestran signos de desgaste, por lo que, en principio, no parece que hayan sufrido procesos previos importantes de remoción tafonómica (sensu Fernández López, 2000). Por el contrario, son frecuentes en los ejemplares de los afloramientos de Ardanatz y Aranguren señales superficiales de disolución. Los pequeños regueros de escorrentía que cortan al regolito permiten mostrar las margas en su posición original y, en algún caso, se observa cómo los fósiles de $R$. spirulaea se disponen verticalmente en relación al plano de estratificación (Fig. 2c).

Debido a la mayor fragilidad de la base de la parte desenrollada del tubo (chimenea), la mayoría de los ejemplares de $R$. spirulaea de los afloramientos prospectados están desprovistos de ésta. Como consecuencia, son frecuentes los fragmentos de chimenea sueltos en el sedimento. Con la excepción de dos ejemplares del valle de Itzagaondoa (Margas de Ilundain), que presentan un pequeño fragmento de molusco bivalvo y de tubo de anélido incrustantes, respectivamente, en los demás fósiles estudiados no se han encontrado evidencias de epibiontes. Respecto a estructuras bioerosivas, tan solo las hay producidas por briozoos (? Pinaceocladichnus isp.) en tres ejemplares de Ardanatz-Eguesibar y Aranguren (Areniscas de Ardanatz) y por gasterópodos (Oichnus isp.) en seis de la sección de Itzagaondoa (Figs. 2d-e).

\subsection{Rellenos y fosildiagénesis}

En la práctica totalidad de los ejemplares estudiados en lámina delgada, se reconoce un relleno parcial del lumen por un fino sedimento siliciclástico, con un alto componente carbonatado de cristales de micrita-microesparita, 




Figura 6. Microestructura del tubo de un ejemplar (IV.49) de Rotularia spirulaea (Lamarck, 1818) en sección ecuatorial, de la sección de Itzagaondoa (Formación Margas de Ilundain, Bartoniense-(?) Priaboniense de Navarra). a) Vista completa de la sección donde se aprecia la parte enrollada y el paso a la parte terminal no enrollada del tubo. Los diferentes tonos remarcan la zona interna (i), externa (e), transición (t) que constituyen la capa externa (1) y el relleno mayoritario por cemento espático (c). La capa interna (2) no es perceptible a esta escala. Nícoles paralelos. b) Vista parcial del tubo en espiral con su parte más interna de anclaje (a) y el espacio correspondiente quizás a algún fragmento orgánico. En el desarrollo de la espira se puede apreciar, en la parte central, la delgadez de la capa externa (1) con zona interna (i) y zona externa (e) para progresivamente hacerse más gruesa. No es posible reconocer la finísima capa interna (2). A destacar que apenas hay relleno sedimentario frente a la precipitación de calcita equant como cemento (c). Se constata un claro criterio de polaridad, dado que en la parte baja/inferior de cada vuelta se acumula el sedimento e incluso se produce acumulación de menas opacas (m). c) Detalle de la primera (1), segunda (2) y tercera (3) vuelta de espira con el relleno de un fino sedimento con microesparita y formación de mena opaca en la zona más baja de cada vuelta. El criterio de polaridad se confirma en la base de las tres vueltas de la espira. Son perceptibles unas muy delgadas líneas oscuras en todas las zonas de la cavidad cementada por calcita (señaladas por flechas negras), estructuras filamentosas, posiblemente de origen microbiano (e.f.), donde se inicia una parte de la nucleación de los cristales de calcita. Nícoles paralelos. d) Misma imagen que en (c) con nícoles cruzados. Detalle mediante MEB de pirita framboidal, reconocida en el relleno de cemento espático. 
reconocidos por la tinción efectuada. Los terrígenos, más visibles por su mayor tamaño $(<50 \mu \mathrm{m})$, son cuarzos (confirmado por DRX), redondeados sin llegar a estar granosoportados, y sin marcar laminaciones. Ocasionalmente se puede reconocer una mayor concentración en la abertura que pudiera taponar la misma, remarcada con una cementación más intensa justo en el contacto inferior con la calcita equant (Fig. 5a). También es destacable una muy delgada franja de óxidos en la zona externa del sedimento, indicativa de aguas oxidantes que permiten precipitar el hierro (en forma de óxidos), cuando se está produciendo la exhumación de la columna litológica.

Respecto a la intensa cementación por calcita equant en el lumen, es apenas visible una etapa incipiente asociada a la capa interna (2) del tubo, con cristales aciculares de calcita muy finos que no llegan a ser continuos a lo largo de la espira. Esta etapa de cementación es relevada por otra mayoritaria que ocupa la totalidad del volumen y se caracteriza por grandes cristales de calcita equant $(100 \mu \mathrm{m})$ que pasan a sparry $(300 \mu \mathrm{m})$ con una disposición radiaxial o como mosaico de calcita radiaxial fibrosa (Figs. 6a-d, 8d-e). En el ejemplar IV.49, se reconoce una polaridad, puesto que restos de sedimento y pequeños cristales de menas opacas (pirita framboidal) se concentran en la misma situación en las tres vueltas de la espira reconocidas (1, 2 y 3). También son visibles en los cristales de calcita cementantes unas estructuras filamentosas (e.f.) muy delgadas y oscuras que pudiera ser de origen orgánico (microbial structures), a partir de las cuales se produce una pequeña nucleación de calcita equant. Esta situación es visible en la parte basal de los tres anillos (Figs. 6a-d).

El estudio del relleno siliciclástico y los cementos carbonatados mediante CL nos aporta la visión de que el relleno aparentemente detrítico tiene un alto contenido en carbonato y resulta luminiscente en tonos rojos no intensos, mientras que algunos cristales de cuarzo detrítico, incluidos

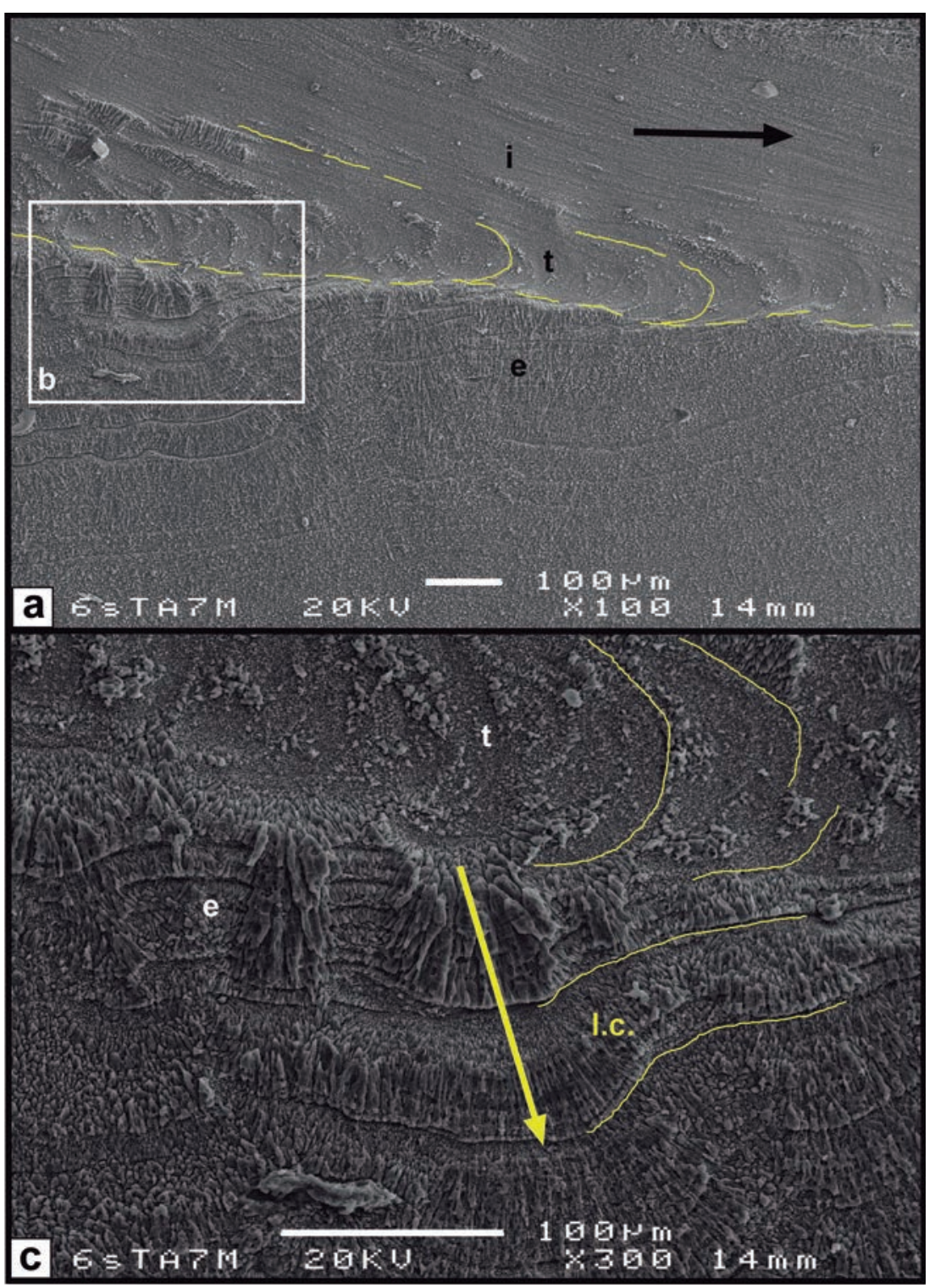

Figura 7. Vista general y de detalle mediante el MEB, de la microestructura del tubo de un ejemplar (AD3.1.19, sección AD3, Ardanatz-Eguesibar) en corte ecuatorial de Rotularia spirulaea (Lamarck, 1818). a) Aspecto general de la capa externa donde se aprecia las líneas de crecimiento de la zona interna (i), zona de transición flexionada (t) y la zona interna (e). El tipo de microestructura homogénea parece ser prismático con los prismas muy finos en (i), pero con un fuerte aumento de tamaño en la (e), remarcada por la disposición esferulítica que adoptan los prismas, separados por las líneas de crecimiento. La zona flexionada corresponde a la transición ( $\mathrm{t}$ ), mostrando la continuidad entre las tres zonas. La flecha negra indica la dirección de enrollamiento. b) Parte seleccionada de la siguiente imagen. c) Detalle de la figura (b) anterior, donde se aprecia la flexión de la zona de transición ( $\mathrm{t}$ ) y el desarrollo de los prismas, con una marcada polaridad en su aumento de grosor (flecha amarilla), conformando una estructura prismática esferulítica limitada por líneas de crecimiento (l.c.) mayores, remarcadas en amarillo. 
en él, se reconocen por la coloración azulada (Figs. 9d, 9h). Respecto a los cementos carbonatados su comportamiento mayoritario es apenas luminiscente (dull) o incluso no luminiscente, tanto los delgados cristales tempranos aciculares y circungranulares (c1) como los considerados radiaxiales (c2). Por último, ocupando espacios centrales en las secciones del lumen de algunos ejemplares del valle de Itzagaondoa aparecen cristales equant con una luminiscencia amarilla intensa, indicativa del estadio final de cementación (c3), solamente reconocidos por su comportamiento luminiscente diferente, al quedar agotado el hierro inhibidor en un sistema ya cerrado (Fig. 9b).

\section{PALEOECOLOGÍA. HÁBITO DE VIDA DE ROTULARIA SPIRULAEA}

\subsection{Interpretaciones previas}

Una parte de los autores consultados consideran que las especies de Rotularia corresponden a organismos de vida bentónica libre, que se disponían tendidos sobre la superficie fangosa y se alimentaban de sedimento (sedimentívoros). Según Savazzi (1995), ejemplares de $R$. spirulaea muestran incrustaciones de hidrozoos $\mathrm{y} / \mathrm{o}$ briozoos en uno o ambos lados, sugiriendo que su tubo enrollado se habría dispuesto tendido en posición subhorizontal sobre la superficie del sedimento, con un lado expuesto pero con frecuentes cambios de posición, en un sedimento tipo calcirrudita o calcarenita con cantidades variables de fango, indicando un ambiente de alta energía en aguas someras. Según este autor, el engrosamiento del tubo de $R$. spirulaea podría ser una adaptación tanto para resistir los impactos de las olas como para reducir la depredación de los organismos trituradores y perforadores de conchas. La forma desenrollada de la última espira sugeriría que Rotularia fue una forma sésil ó escasamente móvil. La carena o carenas periféricas de Rotularia incrementarían la superficie de descanso, aumentando la estabilidad del tubo enrollado y reduciendo probablemente el riesgo de enterrarse en el substrato. Este carácter en Rotularia no alcanzaría la extensión desplegada en bivalvos y gasterópodos, con adaptaciones tipo raqueta de nieve (snow-shoe). Los ejemplares de Rotularia recogidos en las arcillas de Possagno (Italia) por Savazzi (1995) son más pequeños (aproximadamente la mitad de su diámetro) que los provenientes de otros sedimentos más groseros. Además, son mucho menos frecuentes y le sugieren al mencionado autor que el fino barro representa un sustrato menos adecuado para el crecimiento de estos organismos.

En la literatura también se recogen propuestas opuestas a la comentada anteriormente para Rotularia. Así, Macellari (1984), en un estudio sobre cuatro especies del género Rotularia (subgénero Austrorotularia Macellari, 1984) de la Antártida, señala que la presencia del largo tubo final en la mayoría de los ejemplares adultos estudiados, así como la ubicación cercana a la apertura del tubo de orificios de depredación producidos probablemente por gasterópodos natícidos (Gastropoda, Naticidae), pueden ser rasgos indicativos de que fueron poliquetos que vivían enterrados verticalmente por debajo de la superficie (organismos infaunales). Por otra parte, y en sentido opuesto a lo considerado por Savazzi (1995), encuentra una clara correlación entre el porcentaje de fango y la abundancia de Rotularia, organismos que serían responsables de la alta bioturbación que a veces podía observarse en el sedimento y que, en principio, no resultaba muy compatible con un modo de alimentación suspensívoro. Sin embargo, Macellari (1984) describe el comportamiento de un poliqueto tubícula actual, Euchone incolor, estudiado por Young \& Rhodas (1971) en Massachussets, que prolifera en facies de arcillaslimos donde los organismos suspensívoros más típicos no pueden sobrevivir. En ese ambiente turbio la capacidad de resistir de la mencionada especie está ligada a la presencia de un tubo que se proyecta por encima de la superficie fangosa del fondo, así como de un complicado sistema de alimentación; mecanismo similar al que pudo ser usado por Rotularia.

Jäger (1993) está en desacuerdo con las ideas de Macellari (1984) y considera como los adultos de Rotularia son sedentarios y se disponen reclinados con la porción enrollada oblicuamente en el sedimento. La generalización de este hábito de vida no es completamente satisfactorio, puesto que la coplanaridad de las regiones enrolladas y no enrolladas del tubo no está explicada satisfactoriamente. En esta situación, morfotipos irregulares como los ilustrados en la figura 8 de Macellari podrían ser la regla, mejor que la excepción. Por otra parte, considera que el modelo de crecimiento de Rotularia, con su porción terminal no enrollada es un sorprendente ejemplo de un programa morfogenético de cuenta regresiva (count-down), siguiendo las ideas de Seilacher \& Gunji (1993).

Mención especial merece el trabajo de Seilacher et al. (2008) sobre los tubos de gusanos serpúlidos y sabellidos convertidos en moradores secundarios de sedimentos fangosos blandos, procedentes de diferentes lugares. Particularmente, es preciso mencionar la visión ofrecida sobre la posición y modo de vida de las Rotularias del Cretácico Superior y del Eoceno recogidas en la isla de Seymour y otras islas del norte de la península Antártica. Estos autores coinciden con Macellari (1984) en las apreciaciones sobre el dominio del enrollamiento levógiro frente al dextrógiro, su considerable tamaño, el pesado esqueleto, las costillas nudosas en espiral y la forma lenticular que hace a Rotularia apta para un descanso pasivo, reclinado, sobre el fondo durante un estadio de su ontogenia. Sin embargo, los ejemplares 


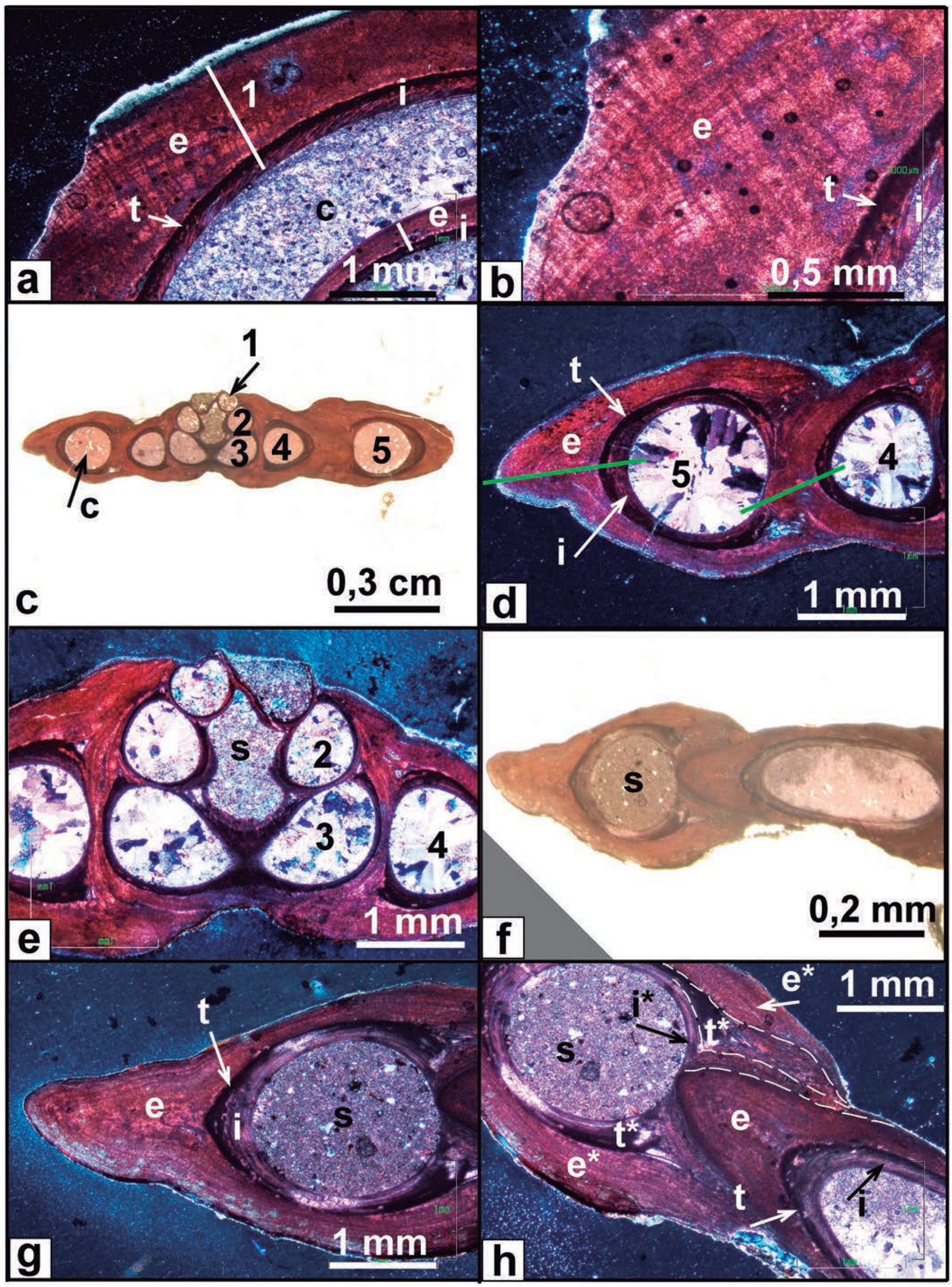


adultos desarrollan una apreciable chimenea siguiendo el plano ecuatorial de la espira, en lugar de elevarse del fondo, tal como la cabeza de una serpiente cobra se eleva sobre su cuerpo enroscado en el suelo. Esta disposición contradice el paradigma funcional de un suspensívoro de modo de vida reclinado sobre el sustrato, propuesto por Savazzi (1995) para Rotularia. Por otro lado, Seilacher et al. (2008) indican la presencia de conchas aisladas dispersas en los sedimentos finos en posición vertical, con la chimenea apuntando hacia arriba. Este hábito de vida podría solamente ser conseguido por una verticalización en la etapa adulta debido a una "active resurrection". En resumen, consideran verosímil que la posición estable reclinada sobre el fondo blando fue solamente un estadio inicial en una secuencia seguida por una verticalización en el estadio adulto, mediante el opérculo tentacular extensible que ha debido ser fundamental en este proceso. Así, su última posición de vida debió de estar cercana a la vertical, con la apertura por encima del fondo blando. La disposición de perforaciones producidas por gasterópodos (Oichnus isp.) en la parte alta de la concha de algunos ejemplares, reafirmaría esta idea, mientras que las concentraciones de conchas de Rotularia sin chimenea y dispuestas horizontalmente como lag deposits serían probablemente el resultado de procesos de remoción post mortem. Posteriormente Seilacher \& Gishlick (2014), en el apartado 21.4 "Rotularia: Countdown Programs for
Resurrection" de su libro Morphodynamics, mantienen esta misma idea.

\subsection{Rotularia spirulaea en la Cuenca de Pamplona}

La interpretación sobre el modo de vida de Rotularia spirulaea aquí propuesta viene a ser coincidente en líneas generales con la de autores como Macellari (1984) y Seilacher et al. (2008). Del análisis efectuado se puede inferir que los ejemplares de $R$. spirulaea debieron adherirse en un primer estadio larvario a pequeños restos orgánicos presentes en el medio, bien en suspensión o ya en el mismo fondo marino fangoso. Se descarta la fijación sobre fragmentos de bioclastos o terrígenos por tres motivos: 1) la ausencia de fragmentos de bioclastos/ terrígenos mayores de 500-800 $\mu \mathrm{m}$ en el homogéneo sedimento margoso (host-rock). Ni siquiera se dan con estos tamaños en el relleno del tubo de Rotularia, donde las partículas son normalmente más gruesas pero inferiores a $50 \mu \mathrm{m}$; 2) en los 523 ejemplares recogidos no se ha reconocido ningún resto cementado en el vértice de la concha; y 3) la forma y tamaño del espacio vacío de adhesión es semejante y todas las conchas poseen una completa perforación umbilical (Ball, 1960; Seilacher et al., 2008). Todo ello parece sugerir que debió ser un

Figura 8. Detalles de la microestructura de los tubos de tres ejemplares de Rotularia spirulaea (Lamarck, 1818) en sección ecuatorial (IV.50, sección de Itzagaondoa, parte alta de la Formación Margas de Ilundain, Bartoniense-(?) Priaboniense, Navarra) y transversales (AG1.4.7; sección AG1 de Aranguren; AD1.3.17; sección AD1 de Ardanatz-Eguesibar, transición entre las formaciones Areniscas de Ardanatz y Margas de Ilundain, Bartoniense, Navarra). a) IV.50, sección ecuatorial parcial de las últimas vueltas de espira, donde la zona externa (e) muestra laminaciones en sentido contrario a las que dibuja la zona interna (i), ambas correspondientes a la capa externa (1). Nótese el mayor grosor tanto de la (i) y (e) de la última vuelta frente a la delgadez de estas mismas zonas en la vuelta anterior. La cavidad queda cementada por calcita equant (c). Nícoles cruzados. b) Detalle de la imagen anterior, con las laminaciones alternantes claras y oscuras en la zona externa (e), la zona de transición (t) y el paso a la zona interna (i). En la zona externa se aprecian bandas espaciadas oscuras perpendiculares a las laminaciones primitivas, indicativas de la microestructura prismática con tendencia esferulítica confirmada por SEM. c) Sección transversal completa de $\mathrm{AD} 1.3 .17$, donde se aprecia un primer estadio trocoespiral ( $1^{\mathrm{a}}-3^{\mathrm{a}}$ vueltas de espira) y otro posterior planiespiral ( $4^{\mathrm{a}}-5^{\mathrm{a}}$ vuelta de espira). Los diferentes tonos son indicativos de la zona interna, externa y la zona de transición más oscura. La cavidad del tubo está completamente rellena de calcita, mientras que la zona de anclaje está ocupada por sedimento (c). Nícoles paralelos. d) Detalle de la imagen anterior con la zona interna (i), externa (e) y la zona de transición $(\mathrm{t})$ bien representadas en el estadio planiespiral ( $4^{\mathrm{a}}$ y $5^{\mathrm{a}}$ vuelta de espira). El plano de simetría de última carena muestra una orientación diferente respecto a la anterior (señalado por las líneas verdes), en un intento de adecuarse a la vertical. La cementación del lumen está exclusivamente producida por calcita radiaxial. e) Detalle de la sección transversal en su desarrollo trocoespiral del mismo ejemplar ( $1^{\mathrm{a}}-3^{\mathrm{a}}$ vueltas $)$ sin carena y la vuelta $4^{\mathrm{a}}$ planiespiral. Se aprecia la zona de anclaje (a) irregular y rota, rellena de sedimento (s). Nícoles cruzados. f) Sección transversal parcial de la parte planiespiral del tubo de AG1.4.7, donde por los diferentes tonos se aprecia la zona interna (i), externa (e) y la zona de transición (t). Nícoles paralelos. g) Detalle de la sección anterior donde es visible la carena terminal constituida enteramente por la zona externa (e), con laminaciones claras y oscuras concéntricas entre sí. Se constata la presencia de la zona de transición (t) y la zona interna (i). La cavidad del tubo está rellenada por un fino sedimento (s). h) Continuación de la sección longitudinal de la imagen anterior hacia la parte central trocoespiral. Se aprecian las mismas microestructuras en la zona interna (i) y externa (e) y el mismo tipo de organización de las laminaciones en la carena más interna. En trazos blancos discontinuos se remarcan los contactos y microestructuras superpuestas a la carena más antigua en el estadio planiespiral, como la zona interior más reciente $\left(i^{*}\right)$, la zona de transición $\left(\mathrm{t}^{*}\right)$ y la zona exterior $\left(\mathrm{t}^{*}\right)$, frente a las más antiguas en el orden de crecimiento continuo de la espira (i), (t) y (e). La capa interna (2) no es perceptible a estos aumentos. 


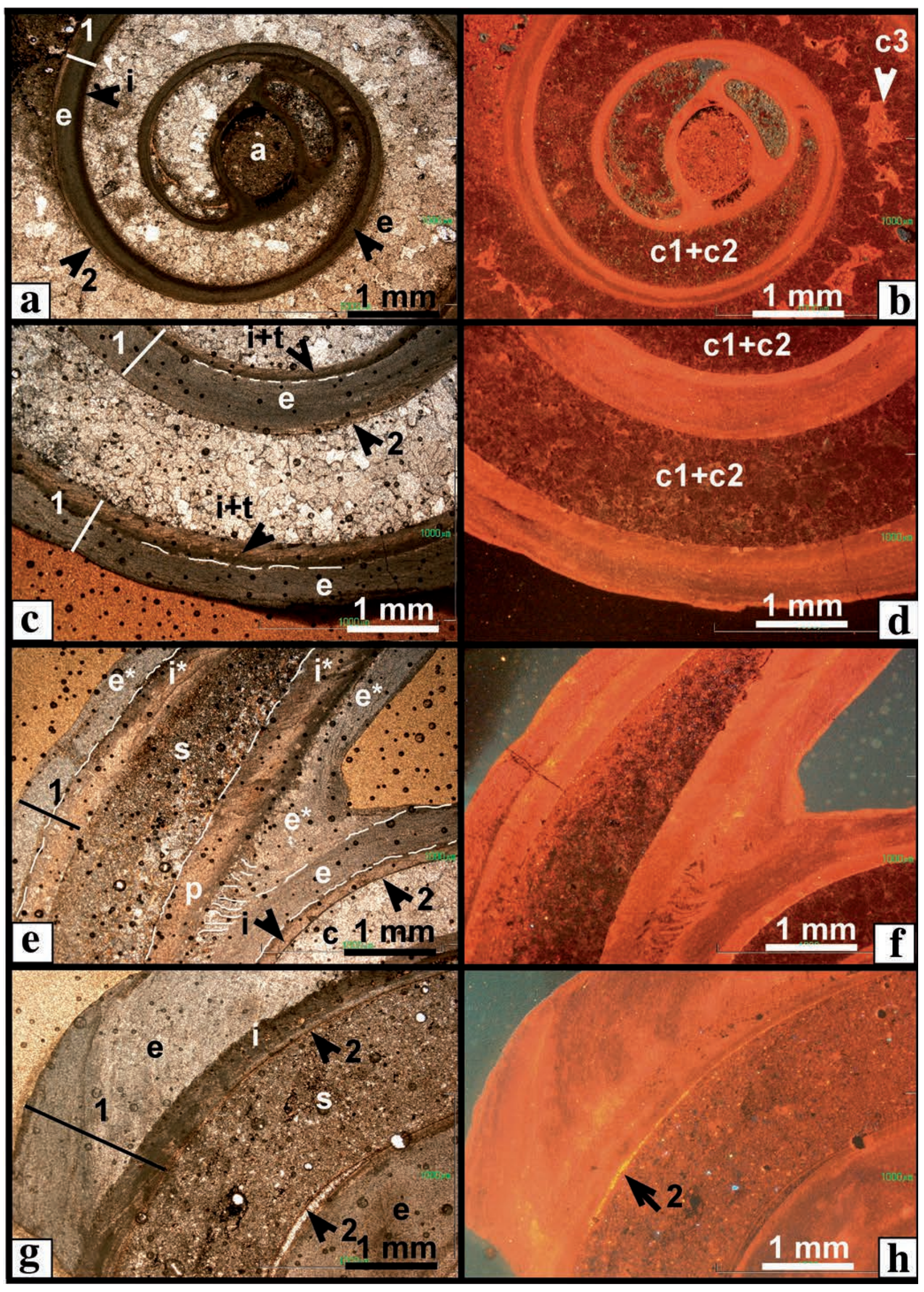


componente orgánico perecedero, de tamaño similar en todos los casos, utilizado en una fase temprana de su vida, sin que la forma exterior de las sucesivas vueltas de espira quedaran condicionadas por la pared de dicho componente. En este sentido, Seilacher et al. (2008) exponen crecimientos de ejemplares de Serpula convoluta del Jurásico medio de Alemania alrededor de tallos cilíndricos perecederos, probablemente pertenecientes a algas marinas.

En una etapa posterior de su ontogenia los individuos quedarían libres sobre el fondo fangoso. A partir de este momento se podrían plantear al menos tres escenarios, según las características del sustrato: 1) un sustrato inestable muy blando donde el ejemplar, a medida que crece se va hundiendo, desarrolla una forma trocoespiral con el vértice apuntando al fondo, sin llegar a desarrollar una chimenea; 2) un sustrato suficientemente estable, que permite una cierta cohesión entre granos, en el que el ejemplar se hace trocoespiral en las primeras vueltas de espira, para más tarde cambiar a planiespiral y, finalmente, poder desarrollar una chimenea; y 3) un sustrato netamente estable, acorde con una ralentización de la sedimentación, que permite una mejor cohesión entre granos, donde el ejemplar se mantiene sin necesidad de producir vueltas trocoespirales, y desarrolla toda su concha como planiespiral con una fina carena hasta alcanzar la madurez con el crecimiento de la chimenea terminal. Es decir, $R$. spirulaea en la zona de estudio presentaría una notable plasticidad ecofenotípica en relación con las condiciones de cada ejemplar en el momento de instalarse sobre el sustrato (Fig. 10). Tengamos presente que la parte alta de la Formación Areniscas de Ardanatz más la totalidad de la Formación Margas de Illundain suponen una potencia probablemente superior a los 500 m (Fig. 2b), producto de una mayoritaria decantación de materiales finos (arcillas y carbonatos), pero con una tasa no necesariamente constante, donde se abrían producido paros temporales en la sedimentación, y los consiguientes inicios de cementación cohesionadora con formación de micro-hardgrounds, que permitieron variar las condiciones cohesivas del fondo blando.

Queda como incógnita el determinar el momento en que se inicia el cambio de la posición inicial reclinada a una posición sub-vertical (active resurrection), con el enterramiento consiguiente propugnado (Seilacher et al., 2008; Seilacher \& Gishlick, 2014). Desde un punto de vista morfofuncional cabe interpretar que la aparición de la quilla observada en la $4^{\text {a }}$ vuelta de espira ya planiespiral (Figs. 8c-h), pudiera propiciar este cambio de posición. La carena permitiría por un lado profundizar mejor en el sustrato suficientemente blando, haciendo una función de arado y por otro, incluso podría favorecer su orientación a modo de veleta, para disponerse enfrentado a las corrientes de fondo ricas en nutrientes, suministradoras del alimento necesario. Resulta notable la diferencia de orientación del plano de simetría de la carena formada en la $4^{\text {a }}$ vuelta

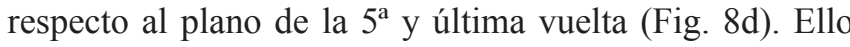
puede ser interpretado cómo que la verticalización no es inmediata y se hace de manera gradual hasta alcanzar el estadio maduro con el desarrollo de la chimenea. El marcado grosor de la capa externa del tubo puede haber realizado una función de lastre en el proceso de verticalización. Por otro lado, la disminución anisométrica de la relación entre diámetro y la altura (espesor) del tubo conforme aumenta la talla (Fig. 4) podría encontrar un significado funcional en el sentido de evitar de este modo una relación superficie/volumen-peso demasiado pequeña para la vida sobre un sustrato blando, en el que los individuos de mayor tamaño podrían hundirse demasiado. Dado que la chimenea se desarrolla en el último estadio de madurez, claramente posterior a las vueltas planiespirales observadas con carena, no parece que esta chimenea pudiera ayudar al proceso de active resurrection. Puede suponerse que la largura de la chimenea estará relacionada directamente con la tasa de sedimentación (a mayor tasa mayor longitud) y también por el gradual hundimiento

Figura 9. Reconocimiento mediante catodoluminiscencia (CL) de las diferentes microestructuras y el relleno del tubo de dos ejemplares (IV.51 y IV.52) de Rotularia spirulaea (Lamarck, 1818) de la sección de Itzagaondoa (Formación Margas de Ilundain, Bartoniense-(?) Priaboniense de Navarra). a) Sección ecuatorial de IV.51 en la zona central de anclaje (a) y las microestructuras interna (i) y externa (e) pertenecientes a la capa externa (1). Es visible también la apenas perceptible capa interna (2). Nícoles paralelos. b) Vista en CL, donde se aprecia la diferencia de luminiscencia entre la (i) y la (e), además de la falta de la misma en el cemento esparítico $(\mathrm{c} 1+\mathrm{c} 2)$, salvo el último estadio (c3) luminiscente. c) Detalle de las dos últimas vueltas de espira de IV.52, donde se aprecia la capa (1) con la zona interna asociada a la zona de transición $(\mathrm{i}+\mathrm{t})$ y externa (e), además de los cementos indiferenciados. Nícoles paralelos. d) Vista en CL, donde la (i+t) unidas son más luminiscentes que la (e). Los cementos $(\mathrm{c} 1+\mathrm{c} 2)$ son apenas luminiscentes. e) Sección de tubo desenrollado donde se aprecia la capa (1) con las zonas interna (i*) y externa ( $\left.\mathrm{e}^{*}\right)$ a ambos lados del mismo frente a la parte enrollada anterior con la (i) y (e), conectadas mediante los pilares (p), además de la finísima capa (2). El relleno del tubo se produce por sedimento (s) y cemento indiferenciado (c). Nícoles paralelos. f) Vista en CL, donde se perciben los comportamientos más luminiscentes de la zona conjunta $(\mathrm{i}+\mathrm{t})$, frente a la menos luminiscente de la zona (e). g) Detalle de la capa externa (1) con la zona interna (i) y externa (e). La capa interna (2) apenas perceptible. h) Vista en CL, donde la capa interna (2) presenta una fuerte luminiscencia amarillo brillante frente a la menor de la capa externa (1) y el relleno del fino sedimento de tonos rojizos-amarillentos. 
en el sedimento menos cohesivo por la forma y peso de cada ejemplar.

Estas ideas, esquematizadas en la Figura 10, sobre el modo de vida infaunal o semi-infaunal en posición vertical de $R$. spirulaea están soportadas, por una parte, por las evidencias encontradas en los afloramientos y, también, por los aspectos texturales que aportan el relleno y el cemento calcítico de la cavidad del tubo. Como se ha comentado, a veces existen acumulaciones de restos de sedimento y minerales opacos (pirita framboidal formada por biodegradación, descomposición de materia orgánica y actividad bacteriana) en las zonas bajas de las vueltas de la espira, indicando que la postura de los organismos enterrados en posición en vida pudiera ser vertical y que una vez muertos se mantenían en el mismo emplazamiento durante al menos parte del tiempo de biodegradación, relleno detrítico y cementación (Figs. 6a-d). Esta disposición de la concha parece coherente con la posición lógica de la zona no enrollada que forma una chimenea señalando la interfase agua-sedimento, además de corroborar con lo reconocido en los afloramientos (Fig. 2c). Un número mayor de observaciones sobre el terreno y bajo el microscopio vendrían a reforzar estas interpretaciones.

Por otra parte, en apoyo de estos planteamientos es de reseñar también que a diferencia de lo indicado por Savazzi (1995) para los fósiles por él estudiados, que frecuentemente presentan hidrozoos y briozoos incrustantes por uno o los dos lados del tubo, en los fósiles de $R$. spirulaea de este trabajo, como ya se ha indicado en el sub-apartado 4.1, prácticamente no hay epibiontes y las señales de bioerosión son escasas en los 523 ejemplares examinados; tan sólo estructuras bioerosivas de briozoos (? Pinaceocladichnus isp.) en tres ejemplares de Ardanatz-Eguesibar y Aranguren (Areniscas de Ardanatz) y orificios de depredación por gasterópodos (Oichnus isp.) en seis ejemplares de Itzagaondoa (Figs. $2 \mathrm{~b}-\mathrm{e})$. La pérdida habitual de la chimenea en la mayoría de los ejemplares pudiera justificarse, además de la fragilidad de la misma, por la posición verticalizada en su último
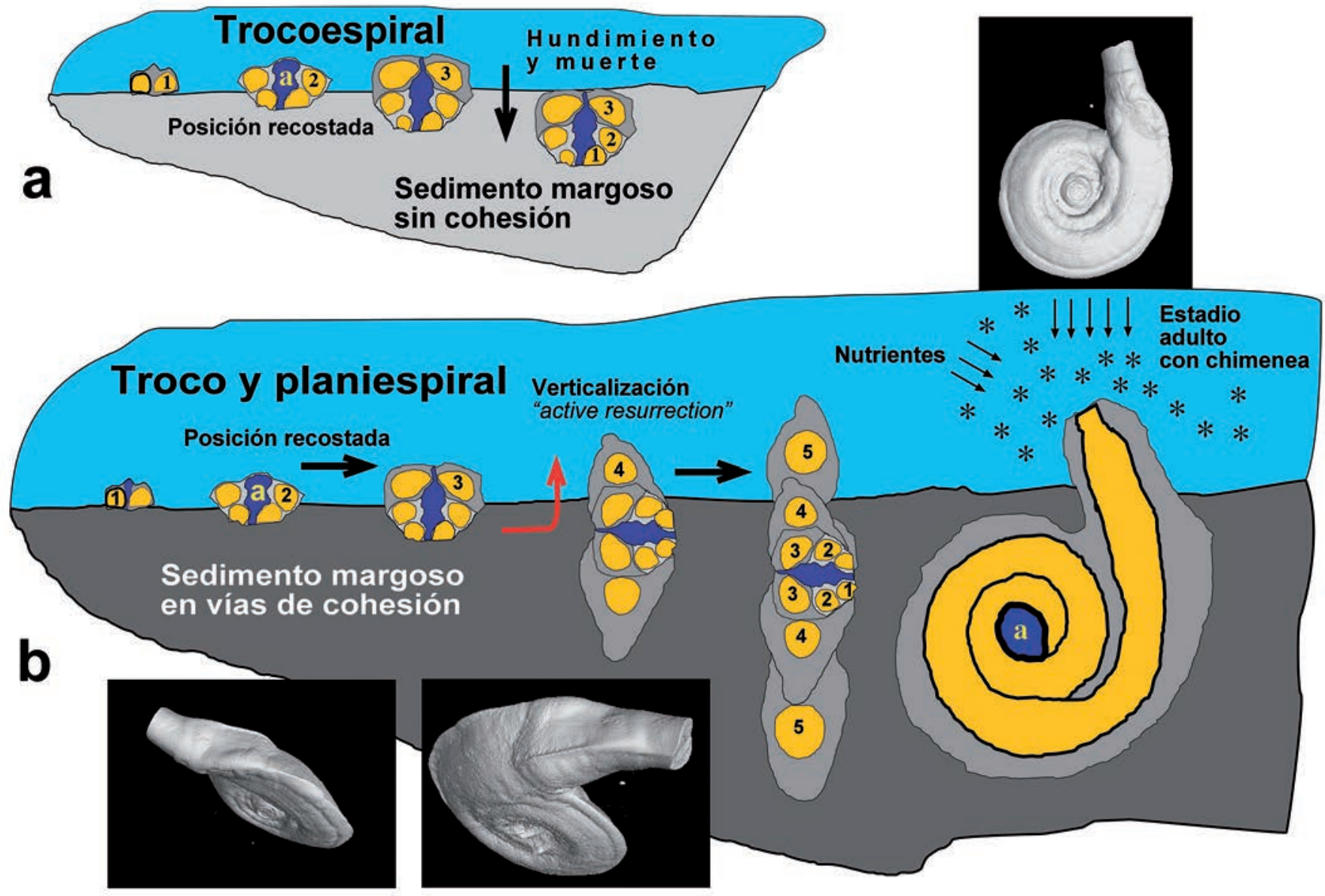

Figura 10. Esquema hipotético de la plasticidad ecofenotípica de Rotularia spirulaea (Lamarck, 1818), según el grado de cohesión del fondo fangoso. a) Desarrollo trocoespiral en un sedimento margoso sin cohesión. b) Cambio de trocoespiral a planiespiral en un sedimento margoso en vías de cohesión, que permite alcanzar el estadio adulto vertical con formación de chimenea. 
estadio, con una cierta inclinación en busca de la posición más adecuada a recibir las corrientes ricas en nutrientes, lo cual permite que la compactación litostática posterior ejerza el suficiente esfuerzo cizallante como para producir su ruptura y pérdida posterior.

\section{CONCLUSIONES}

En este trabajo se reconoce la presencia de numerosos ejemplares sueltos, nunca cementados entre sí, atribuidos a la especie Rotularia spirulaea (Lamarck, 1818), dentro de las formaciones Areniscas de Ardanatz y Margas de Ilundain del Eoceno medio (Bartoniense y (?) Priaboniense) de Navarra.

La composición del tubo fósil de $R$. Spirulaea es calcítica, sin evidencia de la presencia de aragonito. Se confirma que la microestructura del tubo está constituida por dos capas: una externa (1), gruesa creciente (desde $\approx 100$ a $1000 \mu \mathrm{m})$, compuesta por tres zonas en continuidad entre ellas; una interna (i) con una microestructura de muy pequeños cristales, tendentes a ser prismáticos remarcados por la líneas de crecimiento; una zona oscura de transición (t) flexionada y otra zona externa (e), con una estructura prismática con tendencia a esferulítica, pero con la disposición de las líneas de crecimiento en sentido opuesto a las líneas de la zona interna, y otra capa interna (2), de una extrema delgadez $(20-50 \mu \mathrm{m})$, casi imperceptible y siempre delimitando el lumen.

Se propone que el objeto donde se adhiere el organismo en su estado larvario, para estabilizarse en el fondo, fue de naturaleza orgánica y perecedera, por lo que en el vértice del tubo o concha siempre se observa un espacio vacío, muy semejante en tamaño y forma entre los diferentes ejemplares. Por otro lado, la morfología adulta de la concha, con una chimenea que forma un ángulo $\left(<45^{\circ}\right)$ con la parte planiespiral de la misma, las evidencias de campo y del estudio en lámina delgada serían coherentes con un modo de vida infaunal o semi-infaunal y verticalizado de los individuos adultos.

La morfología trocoespiral, trocoespiral-planiespiral y planiespiral de los ejemplares podría estar condicionada por las características del sustrato, sin cohesión, medianamente cohesionado o cohesionado, respectivamente; es decir, por la capacidad de soportar al organismo durante su ontogenia. El tubo presenta un crecimiento anisométrico, haciéndose proporcionalmente más aplanado conforme aumenta el tamaño del mismo, evitando un incremento excesivo de peso que podría resultar perjudicial para su estabilidad en el sedimento La disposición del tubo de $R$. spirulaea durante el estadio trocoespiral sería recostada. Superada esta etapa, podía pasar a una forma planiespiral, con la formación de una carena o quilla estabilizadora.
Esta carena le habría permitido, ayudado por el opérculo tentacular extensible, adquirir, de forma gradual, una posición vertical dentro del sustrato fangoso. A partir de este estadio, se formaría la parte desenrollada del tubo que, a modo de chimenea, sobresaldría parcialmente del sedimento. La cavidad del tubo (lumen) habitualmente está rellenada por sedimento y/o cementada por grandes cristales de calcita, con ejemplos de texturas geopetales, que vendrían a apoyar la idea de una posición de vida vertical en la madurez de los individuos.

\section{AGRADECIMIENTOS}

A los doctores Eduardo Mayoral (Universidad de Huelva, España) y Eduardo B. Olivero (Centro Austral de Investigaciones Científicas, CADIC-CONICET, Argentina), revisores de este trabajo. Sus valiosos comentarios han permitido mejorar apreciablemente el manuscrito. Este trabajo ha sido subvencionado en parte por el Proyecto de Investigación CGL2013-47521-P (Ministerio de Economía y Competitividad, Servicio de Estado de Investigación, Desarrollo e Innovación, MINECO), el Fondo Europeo de Desarrollo Regional (FEDER), los Grupos de Investigación IT1044-16 (Eusko Jaurlaritza/ Gobierno Vasco) y PPG17-05 (Universidad del País Vasco (Euskal Herriko Unibertsitatea, UPV/EHU), y la Fundación Elorza. Los autores agradecen el apoyo técnico y humano de los SGIker de la UPV/EHU; también a la Dra. I. Rosales (IGME, Madrid), por las facilidades recibidas en la utilización del equipo de CL instalado en el IGME.

\section{REFERENCIAS}

Abad, A. 2001. Paleotaxodonta y Pteriomorphia del Eoceno del Margen Sur de la Depresión Catalana. Tesis Doctoral, Universitat Autònoma de Barcelona, Barcelona (inédita). http://ddd.uab.cat/record/37045, 803 pp.

Archiac, A.M. d' 1846. Description des fossiles recueillis par M. Thorent dans les couches à Nummulines des environs de Bayonne. Mémoires de la Société géologique de France, 2ème série, 2, 189-217.

Archiac, A.M. d' 1850. Description des fossiles du groupe nummulitique recueillis par M.S.-P. Pratt et M.J. Delbos aux environs de Bayonne et de Dax. Mémoires de la Société géologique de France, 2ème série, 3, 397-456.

Astibia, H., Murelaga, X., Payros, A., Pereda, X. \& Tosquella, J. 1999. Tortugas y Sirenios fósiles en el Eoceno marino de Navarra y Cuenca de Jaca. Geogaceta, 25, 15-18.

Astibia, H., Payros, A., Pereda Suberbiola, X., Elorza, J., Berreteaga, A., Etxebarria, N., Badiola, A. \& Tosquella, J. 
2005. Sedimentology and taphonomy of sirenian remains from the Middle Eocene of the Pamplona Basin (Navarre, western Pyrenees). Facies, 50, 463-475; doi: 10.1007/ s10347-004-0026-5.

Astibia, H., Pereda Suberbiola, X., Bardet, N., Payros, A., Berreteaga, A. \& Badiola, A. 2006. Nuevos fósiles de sirenios en el Eoceno medio de la Cuenca de Pamplona (Navarra). Revista Española de Paleontología, 21, 79-91.

Astibia, H., Elorza, J., Pisera, A., Álvarez-Pérez, G., Payros, A. \& Ortiz, S. 2014. Sponges and corals from the Middle Eocene (Bartonian) marly formations of the Pamplona Basin (Navarre, western Pyrenees): taphonomy, taxonomy, and paleoenvironments. Facies, 60, 91-110; doi: 10.1007/ s10347-013-0364-2.

Astibia, H., Rodríguez-Tovar, F.J., Díaz-Martínez, I., Payros, A. \& Ortiz, S. 2016a. Trace fossils from the Middle and Upper Eocene (Bartonian-Priabonian) molasse deposits of the Pamplona Basin (Navarre, western Pyrenees): palaeoenvironmental implications. Geological Journal, 52, 327-349; doi: 10.1002/gj.2763.

Astibia, H., Payros, A., Ortiz, S., Elorza, J., Álvarez-Pérez, G., Badiola, A., Bardet, N., Berreteaga, A., Bitner, M.A., Calzada, S., Corral, J.C., Díaz-Martínez, I., Merle, D., Pacaud, J.M., Pereda-Suberbiola, X., Pisera, A., Rodríguez-Tovar, F.J. \& Tosquella, J. 2016b. Fossil associations from the middle and upper Eocene strata of the Pamplona Basin and surrounding areas (Navarre, western Pyrenees). Journal of Iberian Geology, 42, 7-28; doi: 10.5209/rev_JIGE.2016.v42.n1.51601.

Ball, H.W. 1960. Upper Cretaceous Decapoda and Serpulidae from James Ross Island, Graham Land. Falkland Islands Dependencies Survey, Scientific Reports, 24, 1-30.

Barnolas, A., Payros, A., Samso, J.M., Serra-Kiel, J. \& Tosquella, J. 2004. La Cuenca surpirenaica desde el Ilerdiense medio al Priaboniense. In: Geología de España (ed. Vera, J.A.). Instituto Geológico y Minero de España, Sociedad Geológica de España, Madrid, 313-320.

Berkeley, M.J. 1835. Observations upon the Dentalium subulatum of Deshayes. Zoological Journal, London, 5, 424-427.

Bitner, A., Astibia, H. \& Payros, A. 2016. Middle Eocene (Bartonian) brachiopods from the Pamplona Basin, Navarre, South-Western Pyrenees. Batalleria, 23, 1-7.

Bronn, H.G. 1827. Verzeichnis der vom Heidelberger Mineralien-Komptoir verkäuflichen KonchylienPlanzenthier- und anderen Versteinerungen. Zeitschrift für Mineralogie, 2, 529-544.

Bouillé, R. de 1873. Paléontologie de Biarritz et de quelques autres localités des Basses Pyrénées. Memoires de la Première Section, Congrès Scientifique de France, $39^{e}$ Session 1, Pau, 427-468.

Bouillé, R. de 1876. Paléontologie de Biarritz et de quelques autres localités des Basses-Pyrenées. Imprimerie et Lithographie Veronese, Pau, 71 pp.

Buffrénil, V. de, Astibia, H., Pereda Suberbiola, X., Berreteaga, A. \& Bardet, N. 2008. Variation in bone histology of middle Eocene sirenians from Western Europe. Geodiversitas 30, 425-432.
Calzada, S. \& Astibia, H. 1996. Sobre Plicatula pamplonensis Carez: revisión. Bulletin de la Société d'Histoire naturelle de Toulouse, 132, 65-69.

Carez, L. 1881. Étude des Terrains Crétacés et Tertiaires du Nord de l'Espagne. Savy Edit, Paris, 327 pp.

Carter, J.G., Bandel, K., De Buffrénil, V., Carlson, S.J., Castanet, J., Crenshaw, M.A., Dalingwater, J.E., Francillion-Vieillot, H., Géradie, J., Meunier, F.J., Mutvei, H., De Ricqlès, A., Sire, J.Y., Smith, A.B., Wendt, J., Williams, A. \& Zylberberg, L. 1990. Glossary of skeletal biomineralization. In: Skeletal Biomineralization: Patterns, Processes and Evolutionary Trends (ed. Carter, J.G.), Van Nostrand Reinhold, New York. 1, 609-671.

Chamberlin, R.V. 1919. The Annelida Polychaeta, "Albatross" Expedition. Memoirs of the Museum of Comparative Zoology, Harvard, 48, 1-518.

Defrance, M. 1827. Serpule. In: Dictionnaire des Sciences Naturelles (ed. Levrault, F.G.), Le Normant, Strasbourg, Paris. 48, 549-572.

Dickson, J.A.D. 1965. A modified staining technique for carbonates in thin section. Nature, 205, 587. doi: $10.1038 / 205587 \mathrm{a} 0$.

Fauchald, K. 1977. The polychaete worms. Definitions and keys to the orders, families and genera. Natural History Museum of Los Angeles County, Science Series, 28, 1-190.

Fernández López, S.R. 2000. Temas de Tafonomía. Departamento de Paleontología, Universidad Complutense de Madrid, 167 pp.

Fozy, I. \& Szente, I. 2014. Fossils of the Carpathian Region. Indiana University Press, Bloomington and Indianapolis, $508 \mathrm{pp}$.

Gardner, J. 1939. Notes on Fossils from the Eocene of the Gulf Province. l. The Annelid Genus Tubulostium. ll. The Gastropod Families Cassididae, Ficidae, and Buccinidae. U.S. Geological Survey, Professional Paper, 193-B, 17-44.

Grube, A.E. 1850. Die Familien der Anneliden. Archiv für Naturgeschichte, 16, 249-364.

Hosgör, I. \& Okan, Y. 2006. The annelid polychaete Rotularia spirulaea Lamarck, 1818 from the early Middle Eocene (middle-late Cuisian) of Çankırı Basin (Central Anatolia, Turkey). Journal of the Earth Sciences Application and Research Centre of Hacettepe University, 27, 173-179.

Ippolitov, A.P., Vinn, O., Kupriyanova, E.K. \& Jäger, M. 2014. Written in stone: history of serpulid polychaetes through time. Memoirs of Museum Victoria, 71, 123-159.

Jäger, M. 1993. Danian Serpulidae and Spirorbidae from NE Belgium and SE Netherlands: K/T boundary extinction, survival, and origination patterns. Contributions in Tertiary and Quaternary Geology, 29, 73-137.

Jäger, M. 2005. Serpulidae und Spirorbidae (Polychaeta sedentaria) aus Campan und Maastricht von Norddeutschland, den Niederlanden, Belgien und angrenzenden Gebieten. Geologisches Jahrbuch, Reihe A, 157, 121-249.

Lamarck, J.-B. de 1818. Histoire Naturelle des Animaux sans Vertèbres, Présentant les Caractères Généraux et Particuliers de ces Animaux, leur Distribution, leurs Classes, leurs Familles, leurs Genres et la Citation des 
Principales Espèces qui s'y Rapportent, Précédée d'une Introduction Offrant la Détermination des Caractères Essentiels de l'Animal, sa Distinction du Végétal et des Autres Corps Naturels, enfin, l'Exposition des Principes Fondamentaux de la Zoologie, 5. Déterville et Verdière, Paris, $612 \mathrm{p}$.

Lundgren, B. 1891. Stndier öfver fossilförande lösa block. Geologiska Föreningen i Stockholm Förhandlingar, 13, 111-121; doi: 10.1080/11035899109446863.

Macellari, C.E. 1984. Revision of serpulids of the genus Rotularia (Annelida) at Seymour Island (Antarctic peninsula) and their value in stratigraphy. Journal of Paleontology, 58, 1098-1116.

Mendizábal, J. \& Ruiz de Gaona, M. 1949. Memoria Explicativa de la Hoja 141, Pamplona. MAGNA, 1:50.000. Instituto Geológico y Minero de España. Madrid.

Mikuž, V. 2008. The serpulid worm Rotularia spirulaea from Eocene beds near Gracisce in Istria, Croatia. Geologija, 51, 161-168.

Müller, A.H. 1966. Zur Kenntnis mesozoischer Serpuliden (Annelida, Polychaeta). Geologie, 15, 1053-1075.

Oppenheim, P. 1901. Über einige alttertiäre Faunen der Österreichisch-Ungarischen Monarchie. Beiträge zur Paläontologie und Geologie Österreich-Ungarns und des Orients, 13, 145-277.

Payros, A., Pujalte, V., Orue-Etxebarria, X. \& Baceta, J.I. 1997. Un sistema turbidítico Bartoniense de tipo "channel-levee" en la Cuenca de Pamplona: implicaciones tectónicas y paleogeográficas. Geogaceta, 22, 145-148.

Payros, A., Astibia, H., Cearreta, A., Pereda-Suberbiola, X., Murelaga, X. \& Badiola, A. 2000. The Upper Eocene South Pyrenean Coastal Deposits (Liédena Sandstone, Navarre): Sedimentary Facies, Benthic Foraminifera and Avian Ichnology. Facies, 42, 107-132; doi: 10.1007/ BF02562569.

Plaziat, J.C. 1981. Late Cretaceous to Late Eocene paleogeographic evolution of southwest Europe. Palaeogeography, Palaeoclimatolology, Palaeoecology, 36, 263-320; doi: 10.1016/0031-0182(81)90110-3.

Pujalte, V., Baceta, J.I. \& Payros, A. 2002. Tertiary: Western Pyrenees and Basque-Cantabrian region. In: The Geology of Spain (eds. Gibbons, W. \& Moreno, T.). Geological Society of London, London, 293-301.

Puigdefábregas, C. 1975. La sedimentación molásica en la cuenca de Jaca. Pirineos, 104, 1-188.

Rafinesque, C.S. 1815. Analyse de la Nature ou Tableau de l'Universe et des Corps Organisés. J. Barravecchia, Palermo, 223 pp.

Rouault, A. 1850. Description des fossiles du terrain éocène des environs de Pau. Mémoires de la Société géologique de France, 2 ème série, 3, 457-502.

Rouse, G.W. \& Fauchald, K. 1997. Cladistics and polychaetes. Zoologica Scripta, 26, 139-204; doi: 10.1111/j.14636409.1997.tb00412.x.

Ruiz de Gaona, M. 1947. El Bartoniense de la Cuenca de Pamplona. Notas y Comunicaciones del Instituto Geológico y Minero de España, 17, 155-165.
Ruiz de Gaona, M. \& Colom, G. 1950. Estudios sobre las sinecias de los foraminíferos eocénicos de la vertiente meridional del Pirineo (Cataluña-Vizcaya). Estudios Geológicos, 12, 293-434.

Savazzi, E. 1995. Morphology and mode of life of the polychaete Rotularia. Paläontologische Zeitschrift, 69, 73-85; doi: 10.1007/BF02985975.

Schauroth, C.F. von. 1865. Verzeichnis der Versteinerungen im Herzoglichen Naturaliencabinet zu Coburg. Coburg, $327 \mathrm{pp}$.

Schlotheim, E.F. von. 1820. Die Petrefactenkunde auf ihrem jetzigen Standpunkte: durch die Beschreibung seiner Sammlung versteinerter und fossiler Überreste des Thierund Pflanzenreichs der Vorwelt erläutert. Gotha, Becker, $437 \mathrm{pp}$.

Schmidt, W.J. 1955. Nomenklatur und Systematik der Serpuliden-Gattung Rotularia DeFrance (Tubulostium Stoliczka). Mitteilungen der Geologischen Gesellschaft in Wien, 47,159-182.

Seilacher, A. \& Gishlick, A.D. 2014. Morphodynamics. CRC Press. Taylor \& Francis Group, 551 pp.

Seilacher, A. \& Gunji, Y.P. 1993. Morphogenetic countdowns: another view on heteromorph shells in Gastropods and Ammonites. Neues Jahrbuch für Geologie und Paläontologie Abhandlungen, 190, 73-101.

Seilacher, A., Olivero, E.B., Butts, S.H. \& Jäger, M. 2008. Soft-bottom tube worms: from irregular to programmed shell growth. Lethaia, 41, 349-365; doi: 10.1111/j.15023931.2008.00092.x.

Sowerby, J. de C. 1829. The Mineral Conchology of Great Britain VI. London, 230 pp.

Stevens, G.R. 1967. Upper Jurassic fossils from Ellsworth Land, West Antarctica, and notes on Upper Jurassic biogeography of the South Pacific Region. New Zealand Journal of Geology and Geophysics, 10, 345-393.

Stoliczka, F. 1868. The Cretaceous fauna of southern India. Vol. II. The Gastropoda. Palaeontologia Indica, 2, 1-498.

Vinn, O. 2005. The tube ultrastructure of serpulids (Annelida, Polychaeta) Pentaditrupa subtorquata, Cretaeous and Nogrobs cf. vertebralis, Jurassic from Germany. Proceeding of the Estonian Academy of Sciences, Geology, $54,260-265$.

Vinn, O. 2007. Taxonomic implications and fossilization of tube ultrastructure of some Cenozoic serpulids (Annelida, Polychaeta) from Europe. Neues Jahrbuch für Geologie und Paläontologie-Abhandlungen, 244, 115-128; doi: 10.1127/0077-7749/2007/0244-0115.

Vinn, O. 2008. Tube ultrastructure of the fósil genus Rotularia Defrance, 1827 (Polychaeta, Serpulidae). Journal of Paleontology, 82, 206-212; doi: 10.1666/06-125.1.

Vinn, O. \& Kupriyanova, E.K. 2011. Evolution of a dense outer protective tube layer in serpulids (Polychaeta, Annelida). Carnets de Géologie /Notebooks on Geology, Letter 2011/05 (CG2011_L05), Géologie/Notebooks 137-147.

Vinn, O., ten Hove, H.A., Mutvei, H. \& Kirsimäe, K. 2008. Ultraestructure and mineral composition of serpulid 
tubes (Polychaeta, Annelida). Zoological Journal of the Linnean Society, 154, 633-650; doi: 10.1111/j.10963642.2008.00421.x.

Whitfield, R.P. 1890. Observations on some Cretaceous fossils from the Beyrut District of Syria, in the collection of the American Museum of Natural History, with descriptions of some new species. Bulletin of the American Museum of Natural History, 3, 381-441.

Wilckens, O. 1910. Die Anneliden, Bivalven und Gastropoden der antarktischen Kreideformation. Wissenschaftliche Ergebnisse der Schwedische Südpolar Expedition 3, Lief. $12,1-132$.
Wilckens, O. 1922. The Upper Cretaceous Gastropoda of New Zealand. New Zealand Geological Survey Paleontological Bulletin, 9, 42 pp.

Wrigley, A. 1951. Some Eocene serpulids. Proceedings of the Geologists Association of the United Kingdom, 62, 177-197.

Young, D.K. \& Rhoads, D.C. 1971. Animal-sediment relations in Cape Cod Bay, Massachussets. I. A transect study. Marine Biology, 11, 242-254. 